\title{
Differential phase register of Hes1 oscillations with mitoses underlies cell-cycle heterogeneity in $\mathrm{ER}^{+}$ breast cancer cells
}

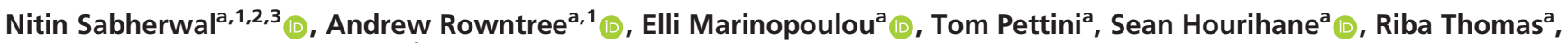 \\ Ximena Soto ${ }^{a}$, Jochen Kursawe ${ }^{b}\left(\mathbb{D}\right.$, and Nancy Papalopulu ${ }^{\text {a, }}$
}

a Division of Developmental Biology and Medicine, School of Medical Sciences, Faculty of Biology, Medicine, and Health, University of Manchester, Manchester M13 9PT, United Kingdom; and ${ }^{\mathrm{b}}$ School of Mathematics and Statistics, University of St Andrews, St Andrews KY16 9SS, United Kingdom

Edited by Ryoichiro Kageyama, RIKEN Noshinkei Kagaku Kenkyu Center, Wako, Japan, and accepted by Editorial Board Member Anton Berns September 8 , 2021 (received for review July 29, 2021)

\begin{abstract}
Here, we study the dynamical expression of endogenously labeled Hes1, a transcriptional repressor implicated in controlling cell proliferation, to understand how cell-cycle length heterogeneity is generated in estrogen receptor (ER) ${ }^{+}$breast cancer cells. We find that Hes 1 shows oscillatory expression with $\sim 25 \mathrm{~h}$ periodicity and during each cell cycle has a variable peak in G1, a trough around G1-S transition, and a less variable second peak in G2/M. Compared to other subpopulations, the cell cycle in $\mathrm{CD} 44^{\mathrm{High}} \mathrm{CD} 24^{\mathrm{Low}}$ cancer stem cells is longest and most variable. Most cells divide around the peak of the Hes 1 expression wave, but preceding mitoses in slow dividing CD $44^{\text {High }}$ CD24 ${ }^{\text {Low }}$ cells appear phase-shifted, resulting in a late-onset Hes1 peak in G1. The position, duration, and shape of this peak, rather than the Hes1 expression levels, are good predictors of cell-cycle length. Diminishing Hes1 oscillations by enforcing sustained expression slows down the cell cycle, impairs proliferation, abolishes the dynamic expression of p21, and increases the percentage of $\mathrm{CD} 44^{\mathrm{High}} \mathrm{CD} 24^{\text {Low }}$ cells. Reciprocally, blocking the cell cycle causes an elongation of Hes 1 periodicity, suggesting a bidirectional interaction of the Hes1 oscillator and the cell cycle. We propose that Hes1 oscillations are functionally important for the efficient progression of the cell cycle and that the position of mitosis in relation to the Hes 1 wave underlies cell-cycle length heterogeneity in cancer cell subpopulations.
\end{abstract}

Hes1 | cell cycle | oscillations | nongenetic heterogeneity | cancer stem cell fate

$\mathbf{M}$ olecular and phenotypic analyses at the single-cell level have revealed a great deal of heterogeneity in genetically identical populations of cells, which were presumed to be homogeneous based on population averaging methods. Such nongenetic heterogeneity is thought to have several potential sources, ranging from transcriptional noise (intrinsic) to variability of exposure to environmental signals (extrinsic). Nongenetic heterogeneity has both benefits and pitfalls for the optimal function of biological systems (1). In cancer, it represents a significant challenge because nongenetic heterogeneity is a crucial factor underlying the differential response to chemotherapy, the emergence of treatment resistance in the absence of new mutations, as well as the disease relapse driven by the reactivation of dormant cancer stem cells $(2,3)$.

At the phenotypic level, nongenetic heterogeneity can manifest itself in the form of the cell-cycle length heterogeneity, which is particularly important in cancer as it may underlie the transition between rapidly dividing, slowly dividing, and quiescent cancer cells, with implications for cancer progression, relapse, and development of resistance to the treatment (4). Thus, there is a need to understand how cell-cycle heterogeneity may be generated within a population of cells at a mechanistic level.

Most molecular studies of heterogeneity have relied on snapshot measurements of populations of cells, where it can be difficult to distinguish the contribution of dynamical gene expression in the time domain versus different levels of expression, which could be relatively stable over time. The contribution of dynamical gene expression in creating heterogeneity has been brought to the forefront by studies showing that important regulatory molecules show oscillatory expression $(5,6)$. Oscillations in gene expression that are asynchronous between cells are not apparent when viewed as a snapshot and, on the whole, are an underappreciated source of nongenetic heterogeneity in a population of cells. The ever-growing list of such molecules includes genes associated with the cell-cycle and the circadian clock, but also genes such as the DNA damage response protein $\mathrm{p} 53$, the proneural protein Ascl1, and the neurogenic ligand $\mathrm{Dl}$, to mention just a few (6). Notable examples are the key transcription factors of the Hes/Her (mammals/zebrafish) family of genes, which have been shown to oscillate synchronously in somitogenesis and asynchronously in neural progenitor cells, and whose oscillations enable the cell-state transition to differentiation (7-11). Among Hes family genes, Hes1 is of particular importance because it has been implicated in

\section{Significance}

Tumors exhibit heterogeneities that are not due to mutations, including cancer stem cells with different potencies. We show that the cancer stem-cell state predisposed to dormancy in vivo has a highly variable and long cell cycle. Using single-cell live imaging for the transcriptional repressor Hes1 (a key molecule in cancer), we show a type of circadian-like oscillatory expression of Hes1 in all cells in the population. The most potent cancer stem cells tend to divide around the trough of the Hes1 oscillatory wave, a feature predictive of a long cell cycle. A concept proposed here is that the position of cell division with respect to the Hes1 wave is predictive of its prospective cell-cycle length and cancer cellular substate.

Author contributions: N.S. and N.P. designed research; N.S., A.R., E.M., T.P., S.H., R.T., and X.S. performed research; N.S., A.R., E.M., T.P., and J.K. contributed new reagents/ analytic tools; N.S., A.R., E.M., T.P., S.H., and X.S. analyzed data; and N.S., A.R., and N.P. wrote the paper.

The authors declare no competing interest.

This article is a PNAS Direct Submission. R.K. is a guest editor invited by the Editorial Board.

This open access article is distributed under Creative Commons AttributionNonCommercial-NoDerivatives License 4.0 (CC BY-NC-ND).

${ }^{1}$ N.S. and A.R. contributed equally to this work.

${ }^{2}$ Present address: Imagen Therapeutics, Manchester M15 6SE, United Kingdom.

${ }^{3}$ To whom correspondence may be addressed. Email: nancy.papalopulu@manchester. ac.uk or Nitin.Sabherwal@imagentherapeutics.com.

This article contains supporting information online at http://www.pnas.org/lookup/ suppl/doi:10.1073/pnas.2113527118/-/DCSupplemental.

Published November 1, 2021. 
controlling proliferation in a cancer context and shown to control (directly or indirectly) the expression of genes involved in the cell cycle. Indeed, Hes1 is known to affect the cell cycle by repressing both activators $(\mathrm{CyD}, \mathrm{CyE}$, and $\mathrm{E} 2 \mathrm{~F})$ and inhibitors (p21 and p27) of the cell cycle, directly or indirectly (12-16). However, how these conflicting activities of Hes1 are incorporated in the cell-cycle machinery to modulate its kinetics are not well understood. It is also not clear whether Hes1 oscillates in a cancer model and, if so, how the oscillatory expression of Hes1 interfaces and possibly controls the cell cycle.

Here, we use an endogenously tagged reporter to ask whether Hes1 oscillates in estrogen receptor $(\mathrm{ER})^{+}$breast cancer cells and, if so, whether it shows a reproducible correlation with the cell cycle. We have chosen to focus on MCF-7 cells, a key cellular model system of $\mathrm{ER}^{+}$breast cancer because it is a type of cancer with long-term relapse, a known clinical problem (17), which may occur at least in part due to nongenetic heterogeneity, cell plasticity, and exit from quiescence at the cellular level. MCF-7 cells are a good model to look at the influence of gene-expression dynamics in $\mathrm{ER}^{+}$breast cancer because they contain well characterized subpopulations of cancer stem cells $\left(\mathrm{ALDH}{ }^{\mathrm{High}}\right.$ and $\mathrm{CD} 44^{\mathrm{High}} \mathrm{C}$ $\mathrm{D} 24^{\mathrm{Low}}$ cells), which differ in their ability for tumor reinitiation and, over time, convert into each other (18).

We report that Hes1 oscillates with an average of 25-h periodicity and this nearly matches the average cell-cycle length of $\sim 24 \mathrm{~h}$ in most of the MCF-7 cells. Through clustering analysis of single-cell dynamic traces of Hes1 over the cell cycle, we found a reproducible relationship between the Hes1 dynamics and the cell cycle, such that in most cells, the preceding division takes place at or near the peak of Hes1 expression. This peak in Hes1 protein expression is then followed by a dip, the onset of which is followed by the G1-S transition in most cells, leading to a second period of increased Hes1 protein concentration before the next division. A minority of the cells show a delayed and prominent Hes1 peak further into the G1 phase, making them appear as if they divided at the trough rather than the peak of Hes1 expression. In such cells, the division can be described as "out of phase" with the Hes1 oscillator. Such cells tend to have longer cell cycles, and remarkably, they are enriched for $\mathrm{CD} 44^{\mathrm{High}} \mathrm{CD} 24^{\mathrm{Low}}$ cancer stem cells, which also show the highest cell-cycle heterogeneity and the highest propensity to switch in or out of phase. Finally, when we experimentally dampened the Hes1 oscillations, the cell cycle slowed down, p21 dynamics were abolished, and the proportion of $\mathrm{CD} 44^{\mathrm{High}} \mathrm{CD} 24^{\mathrm{Low}}$ increased, indicating the functional significance of Hes1 oscillations for an efficient cell-cycle progression and cell fate. Conversely, blocking the cell cycle with $\mathrm{CoCl}_{2}$ elongated the periodicity of Hes1, suggesting a bidirectional coupling of the Hes1 oscillator and the cell cycle.

We conclude that Hes1 dynamics are important for sculpting the cell cycle, such that it occurs efficiently and with little heterogeneity, around a set average cell-cycle length. We propose that differential alignment of Hes1 dynamics with mitoses (i.e., the phase registration) underlies cell-cycle heterogeneity in breast cancer cells and may underlie the propensity of some stem-like cancer cells to become quiescent.

\section{Results}

CRISPR/Cas9-Mediated N-Terminal Tagging of Endogenous Hes1 with mVenus in ER $^{+}$Breast Cancer Cells (MCF-7). To study Hes1 expression dynamics in $\mathrm{ER}^{+}$breast cancer MCF-7 cells at single-cell level and in real-time, we generated an in-frame fusion of mVenus with the endogenous Hes1. A cDNA cassette expressing mVenus was N-terminally fused to the first exon of Hes1 using a CRISPR/ Cas9 genome-editing approach (homology-directed repair, HDR), followed by FACS-based clonal selection and expansion of mVenus ${ }^{+}$ single cells (Materials and Methods and Fig. 1A). After genotyping, a clonal line (hereafter named clone 19/Cl19) was chosen for any further analysis (SI Appendix, Fig. S1 and Movie S1). Cl19 cells were found to be hemizygous for Hes1, with one Hes1 allele having correct insertion of the mVenus cassette and another one knocked out due to insertion of frame-shift mutation after CRISPR reaction. The Cl19 line being hemizygous for Hes1 eliminated any possible interference to the tagged allele by the untagged allele. Cl19 cells recapitulated the endogenous nuclear Hes1 expression and spatial heterogeneity, as assessed by Hes1 immunostaining of parental MCF-7 cells and mVenus-Hes1 snapshots from live-imaging of Cl19 cells (Fig. $1 B-D$ ). During Incucyte-based image analysis, both parental MCF-7 and Cl19 cells exhibited similar growth rates (SI Appendix, Fig. S2), suggesting that knocking out a Hes1 allele in Cl19 cells did not adversely affect the cells.

We then compared protein half-lives of mVenus-Hes1 in Cl19 cells by live-imaging against an HA-tagged Hes1 transfected in parental MCF-7 cells by Western blot analysis, after Cycloheximide $(\mathrm{CHX})$ treatment. Both the endogenous mVenus-Hes1 and the exogenous HA-Hes1 exhibited half-lives of around $4 \mathrm{~h}$, which is longer than the mouse protein (24 to $26 \mathrm{~min}$ ) (19), consistent with the overall increased protein stability in human cells (20). These results suggest that mVenus fusion to endogenous Hes1 does not affect its half-life (SI Appendix, Fig. S3).

The snapshot heterogeneity of Hes1 expression (Fig. $1 B$ and $D)$ may originate (alone or in combination) from two possible scenarios, which are the presence of cells with various stable Hes1 expression levels or a temporally dynamic Hes1 expression, such as random fluctuations or oscillatory protein expression.

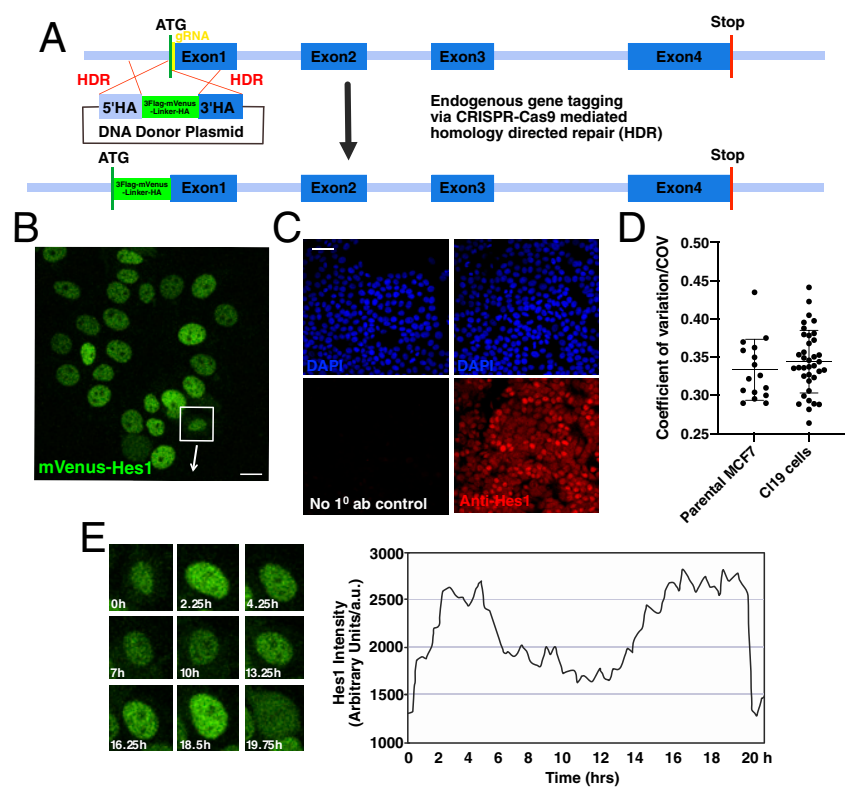

Fig. 1. CRISPR/Cas9-mediated N-terminal tagging of the endogenous human Hes1 with mVenus in MCF-7 cells shows snapshot heterogeneity due to temporal dynamics. (A) Schematic of the Hes1 genomic locus, before and after tagging, the donor plasmid, guide RNA, and how CRISPR/Cas9-mediated HDR reaction achieves in-frame tagging of the first exon of Hes1 with the mVenus CDNA cassette. $(B)$ Imaging of the sequence-verified clonal CRISPR/Cas9 line $(\mathrm{Cl} 19)$ shows nuclear localization of Hes1 and $(C)$ snapshot heterogeneity in the level of endogenous mVenus-Hes1 expression, which were recapitulated by the immuno-staining of parental MCF-7 cells using an anti-Hes1 antibody. (Scale bars, $20 \mu \mathrm{m}$ in $B$ and $50 \mu \mathrm{m}$ in C.) (D) Snapshot heterogeneity as measured by estimating coefficient of variation (COV) from images of $\mathrm{Cl} 19$ cells and Hes1-immunostained MCF-7 cells. COV values (a measure of spatial heterogeneity) showed no differences between parental MCF-7 cells and the CRISPR line (Cl19). (E) Example of a single cell (highlighted with a box in $B$ ) tracked over time. Time-lapse images of this example cell show that Hes1 protein levels fluctuate over time (Left), resulting in a dynamic Hes1 expression as quantified by IMARIS-based cell-tracking (Right). (Magnification, 2X.) 
Single-cell live-imaging over time suggested that the expression of Hes1 is temporally dynamic (Fig. $1 E$ ), which was analyzed further in detail during this study (see Endogenous mVenus-Hes1 Protein Expression in MCF-7 Cells Is Oscillatory with a Circadian-Like Periodicity). To ensure the reproducibility of the dynamics in this clone, we compared it with two other CRISPR/Cas9 clones-one hemizygous (M18) and another heterozygous line (Cl12) - and found them to have similar Hes1 dynamics (SI Appendix, Fig. S4 $A-C)$. No difference in the snapshot heterogeneity in Hes1 expression between the reporter line (Cl19) and the parental MCF-7 cells also suggested that the reporter accurately reflects the endogenous dynamics (Fig. $1 B-D$ ). Thus, population heterogeneity of Hes1 protein expression in $\mathrm{MCF}-7$ is due, at least in part, to temporal Hes1 dynamics, which may be oscillatory, as we assess below (Endogenous mVenus-Hes1 Protein Expression in MCF7 Cells Is Oscillatory with a Circadian-Like Periodicity). Furthermore, single-molecule fluorescence in situ hybridization (smFISH) against Hes1 performed on parental MCF-7 and Cl19 cells showed that number of mature Hes1 mRNA molecules are not constant through the cell cycle, are higher in the G2 phase than in the G1 phase, and suggested some dynamic Hes1 behavior even at mRNA levels both in parental and CRISPRed MCF-7 cells (SI Appendix, Fig. S4D).

MCF-7 Cells Exhibit High Cell-Cycle Length Heterogeneity, Which Is Higher for the Stem Cells. MCF-7 cells in culture are mainly representative of nonstem cells, constituting the majority of the population $(\sim 99 \%)$, referred to as "bulk" cells. A tiny proportion of cells are classed as breast cancer stem cells (bCSCs) in terms of their functional roles in disease progression; CD $44{ }^{\mathrm{High}} \mathrm{CD} 24^{\mathrm{Low}}$ bCSCs tend to be quiescent in vivo, while $\mathrm{ALDH}^{\mathrm{High}}$-type bCSCs are proliferative (18). Therefore, to test whether stem cells might display interesting and useful deviations from the majority of the cells present in culture, we FACS-sorted Cl19 cells into three subpopulations (Materials and Methods) for imaging and analyses. A similar number of cells from each subpopulation $\left(\mathrm{ALDH}^{\mathrm{High}}, \mathrm{CD} 44^{\mathrm{High}} \mathrm{C}\right.$ D2 $4^{\text {Low }}$, and bulk cells) were compared against one another or, if required, pooled together to generate a virtual "mixed" population. Both unsorted and FACS-sorted cellular subpopulations from MCF-7 cells are highly amenable to single-cell live-imaging experiments. We live-imaged subpopulations of cells from Cl19 line for durations encompassing at least one complete cell cycle (mitosis to mitosis). To avoid any effects of synchronizing agents on either the cell-cycle length or the Hes1 expression, we imaged nonsynchronized cells at different stages of their cell cycle. However, later, for data analyses and comparisons, we pseudosynchronized Hes1 time tracks, using the mitoses points as our landmarks.

When characterizing the Hes1 levels and dynamics in relation to the cell cycle, it is crucial to consider the subpopulation heterogeneity that exists in MCF-7 cells. We estimated the cellcycle length from single-cell live-imaging data collected for subpopulations of C119 MCF-7 cells. The reconstituted mixed population data showed a mean cell-cycle length of $29.3 \mathrm{~h}(\mathrm{SD}=$ $11.8 \mathrm{~h}$, median $=25.8 \mathrm{~h}$, interquartile range $[\mathrm{IQR}]=9.6 \mathrm{~h}$ ) with high heterogeneity in the cell-cycle lengths with a heavy-tailed distribution toward longer cell-cycles (Fig. 2A). Bulk cells, which represent the majority of cells in the unsorted, heterogeneous cell population, had a mean cell-cycle length of $23.4 \pm 6.6 \mathrm{~h}$ (mean with SD). By contrast, both stem cell populations had significantly longer cell-cycle lengths, which were on average $29.3 \pm 10.5 \mathrm{~h}$ for $\mathrm{ALDH}^{\mathrm{High}}$ cells and $33.1 \pm 14.3 \mathrm{~h}$ for $\mathrm{CD} 44^{\mathrm{High}} \mathrm{CD} 24^{\mathrm{Low}}$ stem cells (Fig. $2 B$ ). The numbers agree with the preexisting notion that $\mathrm{CD} 44{ }^{\text {High }} \mathrm{CD} 24^{\text {Low }}$ cells represent the quiescent-like stem cells (18), dividing the slowest in normal culture conditions, and ALDH ${ }^{\text {High }}$-type stem cells are more proliferative. In addition, both stem cell populations exhibited higher levels of cell-cycle heterogeneity than the bulk cells, and this was the highest for the $\mathrm{CD} 44^{\mathrm{High}} \mathrm{CD} 24^{\mathrm{Low}}$ population, evidenced by
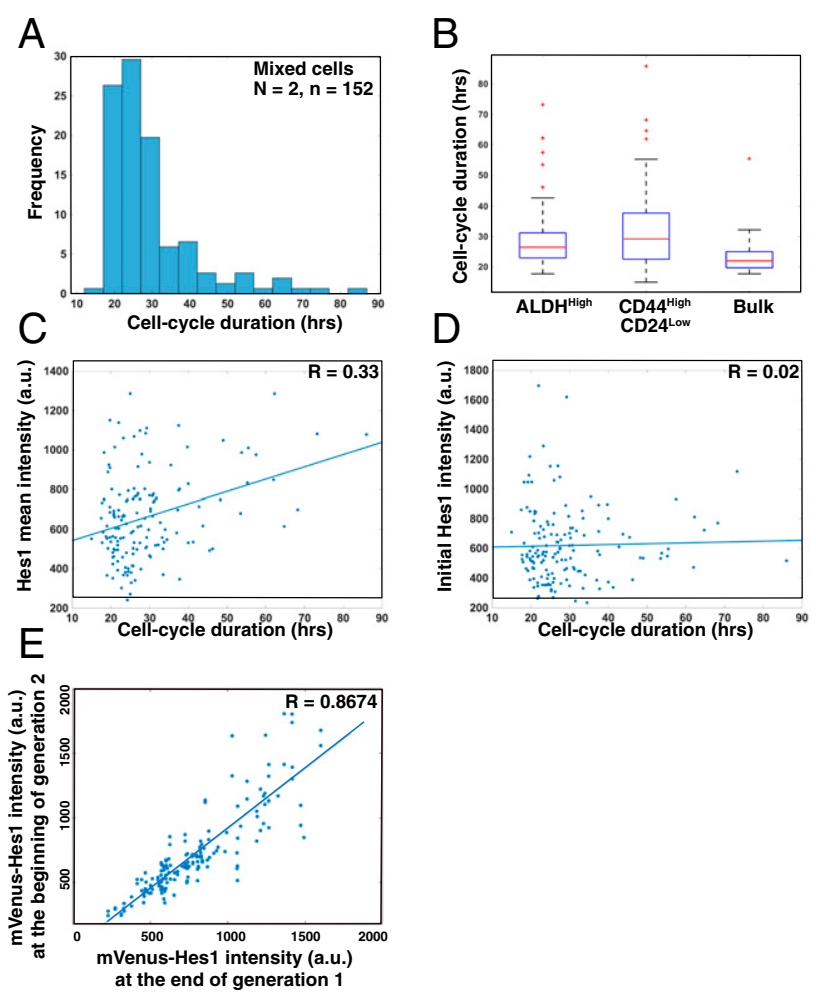

Fig. 2. Cell-cycle length heterogeneity in MCF-7 cells shows no relationship with the Hes1 mean levels. (A) Cell-cycle length of mixed MCF-7 cells has a mean value of around $29 \mathrm{~h}$ but shows high heterogeneity (notice the heavy-tailed distribution of the cell-cycle length). (B) Cell cycle is longer and more heterogeneous for stem-cell subpopulations than bulk cells; $\mathrm{CD} 44^{\mathrm{High}} \mathrm{CD} 24^{\text {Low }}$ cells showed the longest and the most heterogeneous cell cycle. Heterogeneity in these cells was even higher than ALDH ${ }^{\text {High }}$ stem cells with Bartlett's test showing that $\mathrm{CD} 44^{\mathrm{High}} \mathrm{CD} 24^{\text {Low }}$ and $\mathrm{ALDH}{ }^{\mathrm{High}}$ cells have significantly different variances $(* P=0.0192)$. (C) The correlation between the Hes1 mean intensity and the cell-cycle length was overall very modest $(R=0.33)$, as only $11 \%\left(R^{2}=0.11\right)$ of cell-cycle length variance could be attributed to its correlation with the Hes1 levels. $(D)$ The cell-cycle length also did not correlate with the initial Hes1 mean intensity in single cells (immediately after mitoses, in the earlier G1 phases). (E) Mean Hes1 levels do not change immediately prior to and after mitoses. Overall, these data suggest that Hes1 mean expression levels are not a dominant influence on the cell-cycle length and heterogeneity.

the higher "spread" of cell-cycle length values $\left(\mathrm{CD} 44{ }^{\mathrm{High}} \mathrm{C}\right.$ $\mathrm{D} 24^{\mathrm{Low}} \mathrm{IQR}=15.2 \mathrm{~h}, \mathrm{ALDH}^{\mathrm{High}} \mathrm{IQR}=8.25 \mathrm{~h}$, and Bulk $\mathrm{IQR}=5.3 \mathrm{~h})($ Fig. $2 B)$.

The Cell-Cycle Length Shows Very Weak Correlation with the Mean Hes1 Level. Given the significant heterogeneities in cell-cycle lengths among MCF-7 cells, we also asked if there is a correlation between Hes1 expression and the cell cycle that would imply a functional role. We first examined the correlation of Hes1 protein level with the cell-cycle length by plotting the mean Hes1 levels against cell cycle lengths. A Spearman correlation coefficient $(R)$ value of only 0.33 suggested only a very weak correlation between the two (Fig. $2 C$ ); the corresponding $R^{2}$ value of 0.11 would mean that only $11 \%$ of the variance in the cell-cycle length can be attributed to its correlation with the Hes1 mean levels. Furthermore, we found no correlations between the cell-cycle lengths and the initial Hes1 mean intensity (immediately after mitosis) $(R=0.02)$ (Fig. $2 D)$. We also noticed that the mean Hes1 expression in the beginning of the cell cycle is pretty identical to the mean Hes1 expression at the end of the previous cell cycle, meaning there is no sudden loss or gain of Hes1 protein over mitosis (Fig. 2E). This also suggested that the mean Hes1 levels toward the end of previous cell 
A
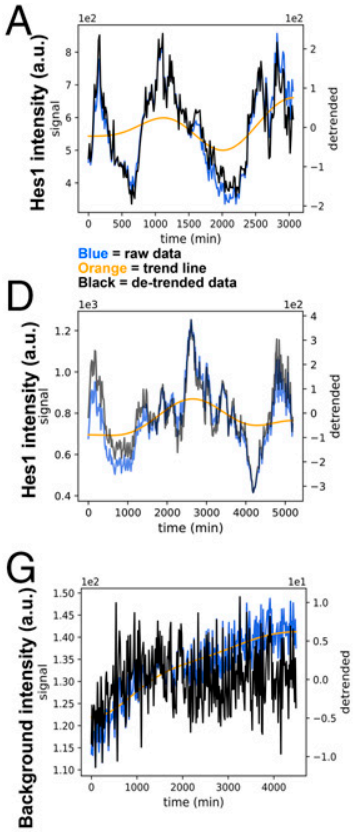
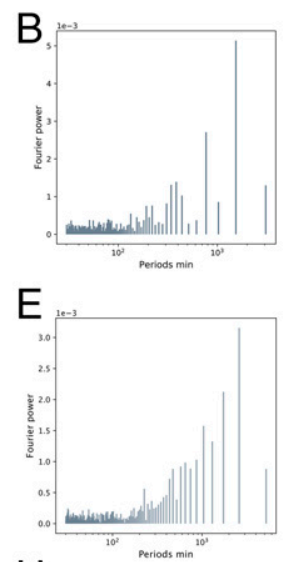

$\mathrm{H}$

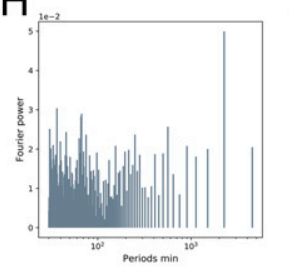

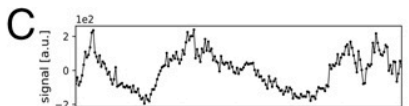

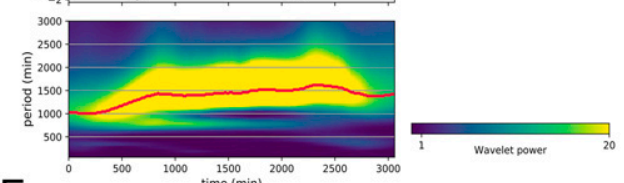

F

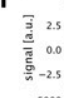

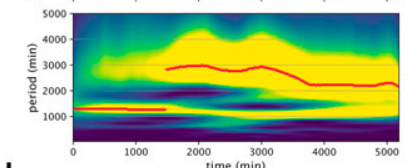

.

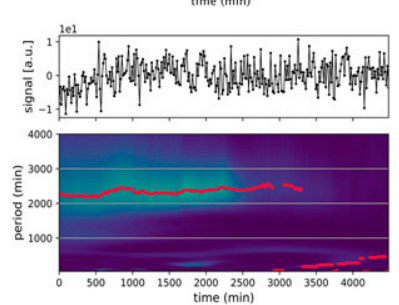

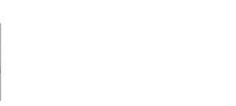
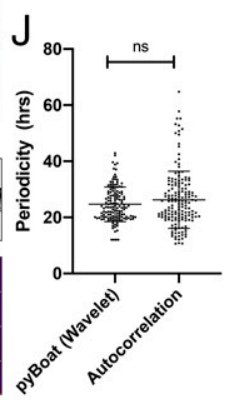

Fig. 3. Wavelet analyses of mVenusHes 1 single-cell time tracks from cellular subpopulation showed evidence for Hes1 wave with a nearly 24-h periodicity. (A) Using the pyBoat platform, mVenus Hes1 time series are detrended prior to periodicity analysis. The black lines show an example of a raw mVenus recorded time series. Blue is the corresponding detrended time series. The orange line denotes the trend. $(B)$ Fourier power spectrum for the same input Hes1 trace shows a dominant period peak at around $25 \mathrm{~h}$. (C) The detrended mVenus-Hes 1 trace is shown again in the Upper panel, with the Lower panel showing the associated period-time-power heat map collapsed in two dimensions. The periods with the highest power at each time point are used to estimate the mean period and power across the duration of the input trace. $(D-F)$ shows similar analyses of another Hes1 trace, which shows elongation of periodicity over time (see red ridge lines in $F$, as compared

to red line in $C)$. (G-I), Fourier and wavelet analysis of a background time series, with no dominant period for the entire track as confirmed through the Fourier power spectrum $(F)$ and the power values at the strongest wavelet periods, which are lower than in $C$. Notice more than 10-fold higher Fourier power for Hes 1 trace in comparison to the background trace ( $F$ vs. $B$ and $E$ ), and also highly coherent period-power values (bright yellow color in $C$ and $F$, shown as a ridge in red) for Hes1, compared to low, noncoherent period-power values for background trace (very low yellow content in $G$ ). ( $J$ ) A mean period of around $25 \mathrm{~h}$ was confirmed in the population through different analyses methods (autocorrelation vs. pyBOAT). ns, not significant.

cycle would not affect the next cell cycle. We thus proceeded by analyzing to what extent dynamics of Hes1 expression, rather than its overall expression level, have an influence on the cell cycle.

Endogenous mVenus-Hes1 Protein Expression in MCF-7 Cells Is 0scillatory with a Circadian-Like Periodicity. We investigated whether Hes1 shows oscillatory expression in MCF-7 subpopulations as has been reported in many developmental systems (8, 9, 21-25). We ran mVenus-Hes1 time traces obtained by stitching together Hes1 dynamics over two consecutive cell divisions, through pyBOAT/Wavelet, a periodicity analyses platform (Fig. 3) (26). The power threshold values for the analyses of actual Hes1 traces (Fig. $3 A-F$ ) were set based on control background traces, which were analyzed similarly but showed no periodicity (Fig. 3 $G-I$ ). Fourier analysis showed that unlike the control traces (Fig. $3 H$ ), mVenus-Hes1 expression showed dominant periods (Fig. 3 $B$ and $E$ ), with mean period around $25 \mathrm{~h}$ (Fig. $3 J)$. In addition to the pyBOAT platform, we also ran our mVenus-Hes1 single-cell time trace data through Lomb-Scargle periodogram (LSP) (SI Appendix, Fig. S5 $A$ and $B$ ) (27). As a biological negative control, we used a viral reporter line-Ubc:NuVenus (expressing mVenus tagged with SV40 nuclear localization signal under the Ubc promoter) - which is not expected to exhibit oscillatory dynamics. LSP analysis showed a sharp peak on the power spectrum for mVenus-Hes1 traces but not for the control (Nu-mVenus) traces (SI Appendix, Fig. S5B); the normalized power values were significantly higher for mVenus-Hes1 than for Nu-mVenus traces (SI Appendix, Fig. S5C). This confirmed that the dynamics of Hes1 protein expression conferred the periodicity observed with the endogenous mVenus-Hes1 reporter. mVenus-Hes1 periodicity analyses with pyBOAT was further corroborated using autocorrelation methods (28), showing a mean value of $\sim 26 \mathrm{~h}$ and similar distributions for Hes1 periodicity values (Fig. $3 J$ ).

As the Hes1 periodicity we report here was higher than the previously reported Hes1 periodicity in mouse cells (ultradian oscillations of 2 to $3 \mathrm{~h}$ ) (23), we looked for evidence of ultradian periodicity in the Hes1 traces in MCF-7 cells using a Gaussian processes-based method (29). Interestingly, an ultradian periodicity of 5 to $6 \mathrm{~h}$ was observed, which was the same in different cellular subtypes but this was embedded within the longer periodicity, giving rise to "nested oscillations" (SI Appendix, Fig. S6 $A$ and $B$ ). None of the features of ultradian oscillations (periodicity, fold change) correlated well with the cell-cycle length (SI Appendix, Fig. S6 $C$ and $D$ ); therefore, we did not follow these further in context of the cell-cycle heterogeneity.

Dynamic Hes1 Expression between Two Consecutive Mitoses Points Is Biphasic. Next, we asked whether the Hes1 dynamic expression has a reproducible relationship with the cell cycle. To facilitate this and for the comparison of large numbers of dynamic Hes1 traces, we visualized all Hes1 traces simultaneously using heatmaps of expression from mitosis to mitosis (Materials and Methods and SI Appendix, Fig. S7). To highlight differences in Hes1 expression dynamics rather than their levels, we normalized Hes1 traces using $z$-scores [(raw intensity - mean intensity)/SD], which enforced that each visualized trace has the same mean and variance. We organized these normalized heat maps in the increasing order of the cell-cycle lengths using the second mitoses as a common end-point (Fig. 4A). We also aligned individual, normalized mVenus-Hes1 time-series traces using the second mitoses as a common end-point (Fig. 4D). Both these alignments showed a biphasic Hes1 expression among all cells, wherein a first Hes1 protein peak was followed by a dip and then by an ascending Hes1 expression toward the end of the cell cycle (Fig. $4 A$ and $D$ ). To ensure that this dynamic behavior was specific to Hes1, we expressed Venus and nuclear Venus under the control of the Ubc constitutive promoter as controls (Fig. $4 B$ and $C$ ). We analyzed their expression traces in the same way as for the mVenus-Hes1. No deducible or reproducible dynamics were observed with either of these controls. 

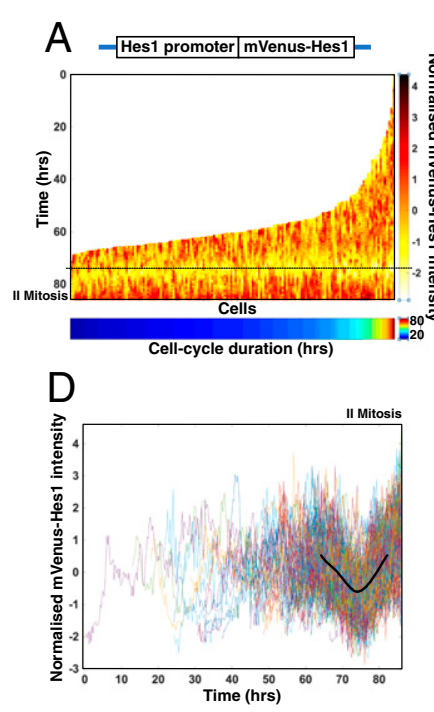

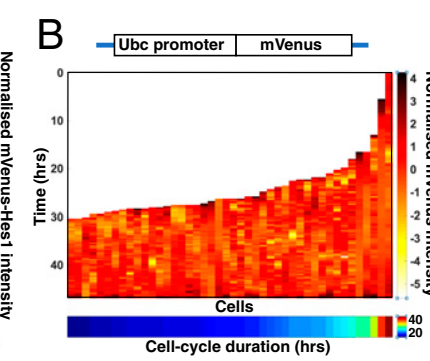

$\mathrm{E}$

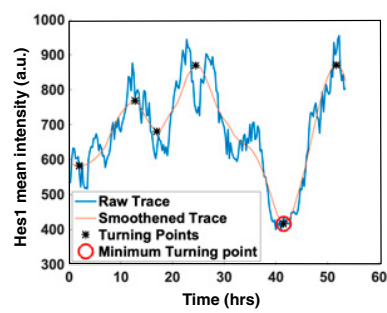

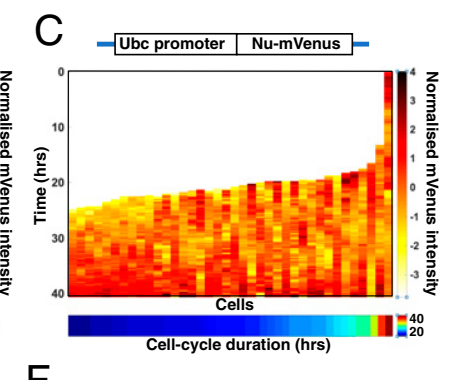

$\mathrm{F}$

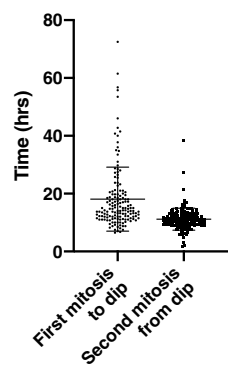

Fig. 4. Dynamic Hes1 expression between two consecutive mitosis points is biphasic. (A) Endogenous mVenus-Hes 1 traces from mixed $\mathrm{Cl} 19$ cells plotted as heat maps (Upper) (SI Appendix, Fig. S7) and organized in increasing order of cell-cycle length (Lower). Notice the presence of the Hes1 expression dip (highlighted by a black line), preceded by a highly variable and followed by a less variable Hes1 peak, giving rise to the biphasic Hes1 profile during the cell cycle. When mVenus (B) or Nu-mVenus $(C)$ were expressed under the Ubc promoter as controls, their expression profiles during the cell cycles showed no such characteristics. (D) When we plotted the same individual Hes 1 tracks (as in $A$ ) as line plots, collapsed at the second mitoses at the right-hand side of the graph, we noticed a similar biphasic Hes1 expression dynamic with a variable, first Hes1 peak, followed by a dip in its expression (again marked by a black line), which was followed by a Hes1 peak toward the end of the cell cycle. (E) A MATLAB-based dipdetection (ADD) method in the mVenus-Hes1 expression profile during the cell cycle detected the minimum turning point (dip) in the Hes1 expression with $92 \%$ accuracy, as compared to manual annotation (SI Appendix, Fig. S6). The duration after the dip (until the second mitosis) showed a tight distribution for all the cells with a mean value of around $11 \mathrm{~h}$, while the duration to the dip (from the first mitosis) showed very high variability (6.5 to 86 h) $(F)$. Together, these data showed that Hes1 expression between two consecutive mitoses is biphasic.

We also developed a MATLAB-based automatic dip detection (ADD) pipeline (Materials and Methods), which detected dips in normalized Hes1 time traces with $92 \%$ accuracy $(4 \%$ false positive and $4 \%$ false negative) (Fig. $4 E$ ). When mVenusHes1 single-cell traces were aligned with the second mitosis as the end-point, the duration of the first episode of Hes1 expression was highly variable, ranging from as short as $6.5 \mathrm{~h}$ to as long as $72.5 \mathrm{~h}($ mean $=18.1 \mathrm{~h}, \mathrm{SD}=11.0 \mathrm{~h}, \mathrm{IQR}=8.1 \mathrm{~h})$. In contrast, the position in the time of the dip in Hes1 relative expression in relation to the second mitosis was highly reproducible, which occurred at around $11 \mathrm{~h}$ after the dip $(\mathrm{SD}=3.8 \mathrm{~h}$ and $\mathrm{IQR}=3.2 \mathrm{~h})($ Fig. $4 A, D$, and $F)$.

The Dip in the Biphasic Hes1 Expression Dynamics Correlates with the G1-S Phase Transition of the Cell Cycle. Next, we wanted to see how Hes1 dynamics might overlap with the cell-cycle phases. Building on from a previous methodology (30), we developed a lentiviral mCherry-proliferating cell nuclear antigen (PCNA) fusion construct as a single-color, texture-based reporter of the cell-cycle kinetics. In brief, cells expressing PCNA show smooth nuclear staining in G1 and G2 phases of the cell cycle, while this nuclear staining picks up a punctate pattern during $\mathrm{S}$ phase, marking DNA replication foci. The PCNA protein spills over the entire cell during mitoses due to the breakdown of the nuclear envelope (Fig. $5 A$ and Movies $\mathrm{S} 2 A$ and $\mathrm{S} 2 B$ ). We performed imaging and analysis on unsorted Cl19 cells virally transduced with mCherry-PCNA so that we could correlate Hes1 dynamics with the cell-cycle kinetics (Fig. $5 A$ and $B$ ). "Congo flag" analysis (Fig. $5 C$ ) showed that in the majority of the cells $(93.3 \%, n=45)$, G1-to-S transition either happened within the Hes1 dip ( $5 \mathrm{~h}$ on either side of the lowest point in Hes1 expression as detected by the ADD pipeline) $(50 \%)$ or after the onset of the Hes1 dip (43.3\%); only a minority of the cells $(6.7 \%)$ showed G1-to-S transition before the appearance of the Hes1 dip. The S-to-G2 transition happened when Hes1 expression started increasing again after the dip in the majority ( $84 \%$ ) of the cells (example shown in Fig. $5 B$ ).
These data from Figs. 4 and 5 suggest that the earlier, variable Hes1 peak takes place within the G1 phase of the cell cycle, that the dip in Hes1 expression dynamics mostly precedes the G1-to-S transition, and the time-invariant, second Hes1 peak happens between mid-late $\mathrm{S}$ and mitosis.

Gaussian Mixture Model Clustering Sorts Hes1 Dynamics into Three Distinct Classes, with Distinct Cell-Cycle Lengths and Cellular Subpopulation Distributions. To understand how Hes1 dynamics interface with the cell-cycle and whether they fall into distinct classes, we "stretched" the Hes1 heatmaps to identical lengths using linear interpolation to represent dynamics at pseudotime scales. This was followed by classifying these Hes1 pseudotime series using the Gaussian mixture model (GMM) clustering methods (Materials and Methods and SI Appendix, Fig. S8) to ask whether Hes1 mitosis-to-mitosis dynamics can be classified into discernible, individual groups. Clustering of stretched Hes1 time-series data showed three distinct dynamical Hes1 behaviors during the cell-cycle progression with the following features (Fig. $6 A$ and $B$ ): cluster 1 with highlow-high, cluster 2 with medium-low-high, and cluster 3 with lowhigh-low-high relative Hes1 expression. These differences in the dynamics of Hes1 traces during the cell cycle were also apparent in unstretched but organized data as per above-described clusters (SI Appendix, Fig. S9). This, along with the analysis of a synthetic control (SI Appendix, Fig. S8), suggested that they are not an artifact of the pseudotime analysis. The clusters were also evident when the traces were averaged (Fig. $6 B$ ). Further analyses showed that clusters 1 and 2 had similar cell-cycle lengths (mean length $=\sim 25$ h) and were both enriched in ALDH ${ }^{\text {High }}$ bCSCs (Fig. $6 C$ and $D$ ). Interestingly, cluster 3 was enriched in $\mathrm{CD} 44^{\mathrm{High}} \mathrm{CD} 24^{\mathrm{Low}}$ bCSCs (Fig. $6 C$ and $D$ ) with highly heterogeneous cell-cycle lengths of up to $90 \mathrm{~h}$ (mean length $45.2 \mathrm{~h}$ and IQR $29.5 \mathrm{~h}$ ) (Fig. 6C). We found that cluster 3 cells share these cell-cycle properties with $\mathrm{CD} 44^{\mathrm{High}} \mathrm{C}$ $\mathrm{D} 24^{\mathrm{Low}}$ cells, as also shown in Fig. $2 B$. These data suggest that there are distinct classes of Hes1 dynamics during the cell-cycle and these dynamics are associated with both cellular subtypes and their cell-cycle lengths. 

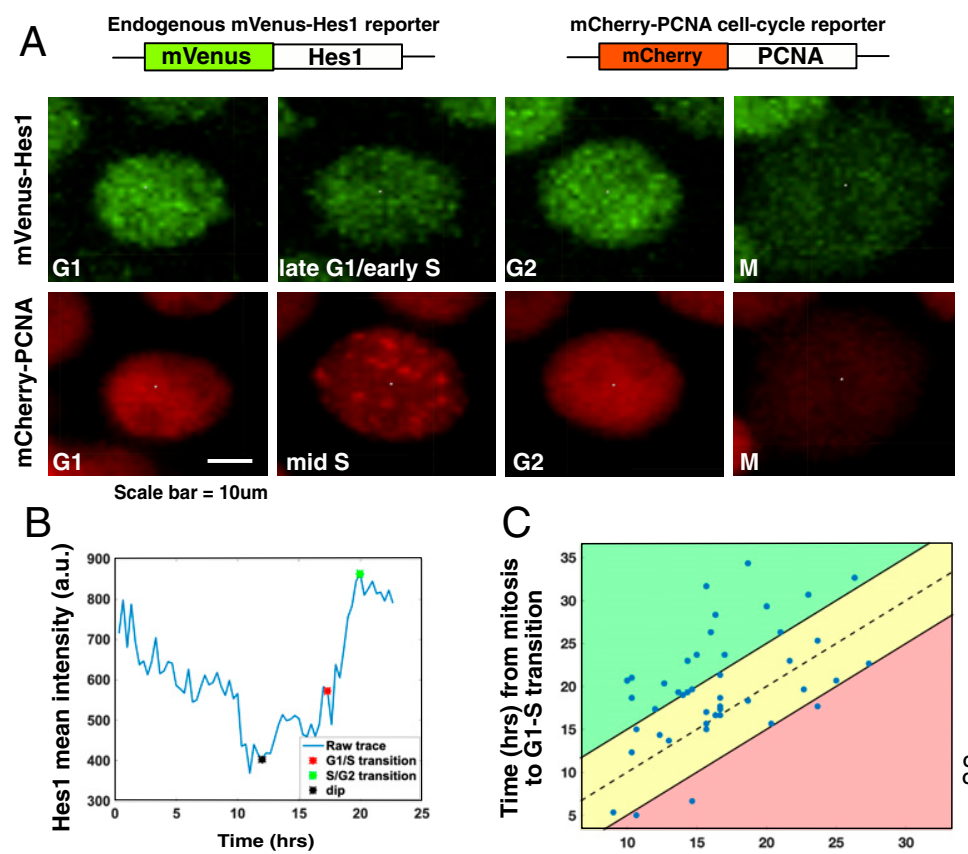

Fig. 5. A PNCA-based live reporter shows that the trough in the biphasic Hes1 expression dynamics coincides with the G1-S phase transition of the cell cycle. (A) Example images of a single cell over time expressing both mVenus-Hes1 and mCherry-PCNA Cl19 unsorted cells were virally transduced with mCherry-PCNA reporter to show how differentially labeled cellcycle phases correlate with the Hes 1 protein expression dynamics. (Scale bar, $10 \mu \mathrm{m}$.) Schematic of Hes 1 and PCNA reporters are shown above the single-cell images. $(B)$ Example of a time trace of Hes1 mean intensity from a single cell expressing PCNA reporter, which was used to mark G1-S and S-G2 transitions, shown as red and green asterisks on top of the Hes1 expression profile. (C) Congo flag plots correlating time from first mitosis to G1-S transition (marked by the onset of appearance of PCNA puncti) with time from mitosis to Hes 1 dip (with the low expression lasting around $10 \mathrm{~h}$, as shown in Fig. 4D) showed that in more than $90 \%$ of the cells the G1-S transition occurs either after the dip in the Hes1 expression (green), during the period of the Hes1 dip (yellow) or at minimum turning point (dotted line), with a small minority of cells showing a G1-S transition before the Hes1 dip (pink).

The Onset of the First Hes1 Peak Correlates with the Cell-Cycle Length. We next sought to understand which feature of the early Hes1 dynamics correlated best with the cell-cycle length. One could hypothesize that cells tend toward a fixed minimum level of Hes1 around G1-S phase transition; therefore, cells with higher peak will take longer to reach this minimum and thus may exhibit a longer cell cycle (Fig. 7A). However, we found no evidence for a defined low level of Hes1 that the cells reached as they went through G1-to-S transition. Instead, a high starting value of Hes1 correlated with a higher Hes1 value at its lowest level, suggesting a constant fold-change. The fold-change in Hes1 expression between the high Hes1 in G1 and low Hes1 at G1-S had a mean value of 1.6. When we plotted Hes1 intensity at G1 peak against Hes1 intensity at the G1-S trough, a strong positive correlation $(R=0.76)$ confirmed the invariability in the Hes1 fold-change (Fig. $7 B$ ); these fold-change values did not show a correlation with the cell-cycle length either $(R=0.10)$ (Fig. $7 C)$. We next looked at the position of the first Hes1 peak by examining the time taken for the cells to reach the first Hes1 peak (Fig. 7D), and found a good positive correlation between time to peak and the cell-cycle length $(R=0.79)$ (Fig. $7 E)$. If we were to remove all values greater than $20 \mathrm{~h}$ on the $y$ axis, we found that the positive correlation still remained but was slightly weaker $(R=0.55)$. We also looked at the area under the curve (AUC) (Fig. 7D) for the first Hes1 peak (as a proxy for the shape of the Hes1 peak), and found an excellent positive correlation between the AUC and the cell-cycle length $(R=0.81)($ Fig. $7 F)$, suggesting that the greater amount of time that Hes1 spends above its lower value correlates with longer cell-cycle length. These findings show that the position and the shape of the Hes1 protein peak within the G1 phase are the best predictors of overall cell-cycle length compared to any other dynamic features.

Mitosis Is Phase-Shifted in Relation to the Hes1 Oscillator in Cells with the Longer Cell Cycle. The late onset of Hes1 expression in cells with longer cell cycle prompted us to examine the phase relations between the mitoses at the beginning of a cell cycle and the Hes1 periodic wave. We constructed phase relation diagrams using Hilbert Transform of the dynamic Hes1 traces using the MATLAB platforms (Fig. $8 A$ ). Phase diagrams for cells (histograms in Fig. $8 B$ and circular plots in Fig. $8 C$ ) suggested that for the majority of cells from clusters 1 and 2 with shorter cell-cycles, the mitoses are distributed around the Hes1 peak and the descending slope toward the Hes1 trough. Since the majority of the cells in the culture divide around the peak (0 and $2 \pi$ ) (Fig. $8 B$, Top), we defined this as the dominant phase ("in-phase") of the Hes1 oscillator and mitosis. Interestingly, cluster 3 cells originate from divisions that preferentially take place at or around a trough of the Hes1 wave (Fig. $8 B$, Middle); therefore, these cells start their cell cycle with a relatively low Hes1, which ascends to an apparently delayed peak of Hes1 expression in G1. We termed this as an "out-of-phase" or "phase-shifted" register of the Hes1 oscillator with mitosis (Fig. 8C and SI Appendix, Fig. S10A). Furthermore, when we presented our data for cellular subpopulations and additionally splitting it into cells with cell-cycle length up to $40 \mathrm{~h}$ and cells with cell-cycle longer than $40 \mathrm{~h}$ (Fig. 8D), it was clear that almost three-quarters of the cells with longer cell-cycle lengths $(>40 \mathrm{~h})$ originated from the mitoses that were out of phase with the Hes1 wave, and that such cells were preferentially of the $\mathrm{CD} 44^{\mathrm{High}} \mathrm{CD} 24^{\text {Low }}$-type (Fig. $8 D$ ). Taken together, these data show that majority of the MCF-7 population exhibit in-phase mitoses around Hes1 peak, resulting in shorter cell cycles. In contrast, out-of-phase mitoses-that is, mitoses occurring closer to a retrospective trough (i.e., one that is only apparent when the next cell division is considered)-result in cells with a longer cell-cycle, which were mainly representing the $\mathrm{CD} 44^{\mathrm{High}} \mathrm{CD} 24^{\mathrm{Low}}$-type cancer stem cells (Fig. $8 C$ and $D$ and SI Appendix, Figs. $\mathrm{S} 10 A$ and $\mathrm{S} 11 D$, right hand side example).

Cancer Stem Cell Subpopulations Differ in Their Switching Probabilities. Cancer stem cell plasticity that underlies nongenetic heterogeneity postulates that cells are not fixed in their properties over time. Therefore, we looked for evidence of switching behavior between clusters 1,2 , and 3 , defined by the Hes1 dynamics over two generations (normalized and clustered separately) (Fig. $9 A$ and SI Appendix, Fig. S10). We found that 
A

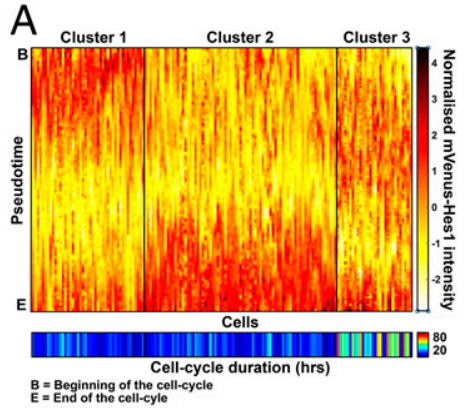

B
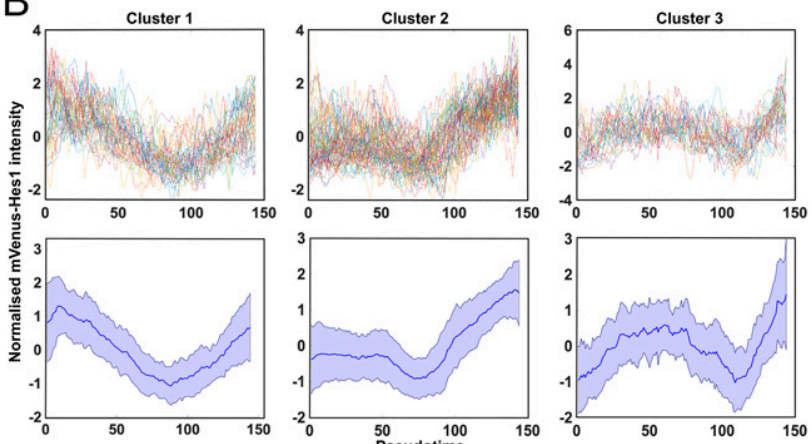

C

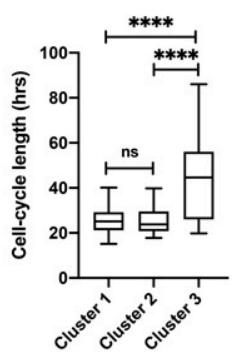

D

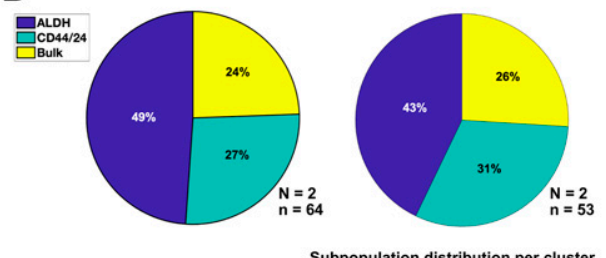

Subpopulation distribution per cluster
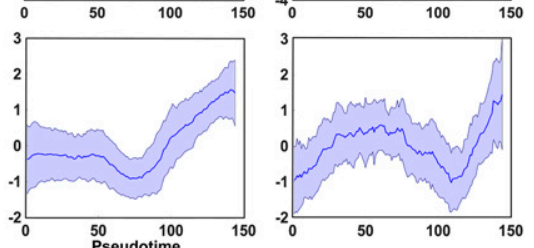

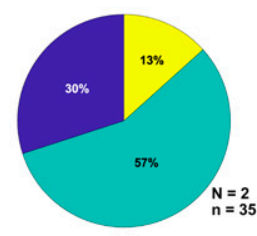

Fig. 6. GMM shows three clusters with distinct Hes1 expression dynamics that map to cellular substates and cell-cycle lengths. (A) GMM clustering classifies Hes1 time traces (stretched from mitosis to mitosis, zscored heat maps) into three distinct classes (clusters 1, 2, and 3, Upper). (Lower) Cell-cycle lengths of individual cells represented as heat maps, showing that cluster 3 is enriched for cells with longer cell-cycles. $(B)$ These clusters can be defined in terms of distinct mean and normalized ( $z$ scores) Hes1 expression dynamics with clusters 1, 2, and 3 having highlow-high, med-low-high, and lowhigh-low-high Hes1 dynamics, respectively, shown by individual (Upper) and averaged (Lower) Hes1 tracks. Dark blue line in the Lower panels show the mean Hes1 behavior per cluster, with lighter blue shading representing the SD errors. (C) Cellcycle length distributions of individual clusters confirm that cluster 3 consists of cells with the longest and the most heterogeneous cell-cycle, while clusters 1 and 2 are enriched for cells with shorter cell-cycles and less heterogeneity (1 vs 2 : ns [not significant], $P=0.6617,1$ vs $3: * * * * P<0.0001,2$ vs $3: * * * * P<0.0001)$. (D) While cluster 3 is enriched for CD44 ${ }^{\text {High }}$ CD24 ${ }^{\text {Low }}$ bCSCs, clusters 1 and 2 are enriched for ALDH ${ }^{\text {High }}$ bCSCs. Overall, Hes1 dynamic behavior can be classified into three classes with distinct cell-cycle lengths and subpopulation distributions.

cells do indeed switch between cluster classifications and these "cluster transitions" are seemingly random: that is, cells from any cluster in generation 1 (mother cell) can fall into any cluster classification upon division into generation 2 (daughter cell) (Fig. 9A). However, they are weighted in that some transitions are more likely than others (e.g., 3 into 1 is more likely than 3 into 3 ). Transition into cluster 3 (out-of-phase behavior) is less frequent in all clusters than transitions into clusters 1 and 2 . Moreover, cells from cluster 3 switche to cluster 1 and 2 more often (i.e., cells with out-of-phase behavior almost invariably divide back into phase) than clusters 1 and 2 that tend to stay in clusters 1 and 2 (Fig. 9B). We also found that cells that have been sorted for $\mathrm{CD} 44^{\mathrm{High}} \mathrm{CD} 24^{\mathrm{Low}}$ cells switch to or from cluster 3 more often than ALDH ${ }^{\text {High }}$ cells. This is consistent with the cluster-switching frequencies above, since cluster 3 is enriched in $\mathrm{CD} 44^{\mathrm{High}} \mathrm{CD} 24^{\mathrm{Low}}$ cells, and $\mathrm{CD} 44^{\mathrm{High}} \mathrm{CD} 24^{\mathrm{Low}}$ cells tend to fall into cluster 3 more often than ALDH ${ }^{\text {high }}$ cells (Fig. 9C). In all cases, transitions into or out of cluster 3 were accompanied by an elongation or a shortening of the cell-cycle length and Hes1 periodicity (SI Appendix, Figs. S10 and S11B), which further strengthened the link of the phase register with the cell-cycle length.

Bidirectional Functional Interaction of the Hes1 Oscillator and the Cell Cycle. To ask whether the influence from Hes1 oscillations to cell-cycle length is causative, we directly manipulated the
A

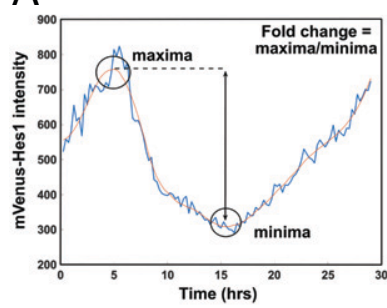

D

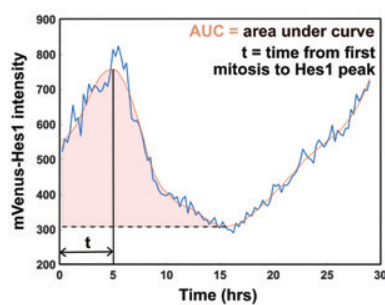

B

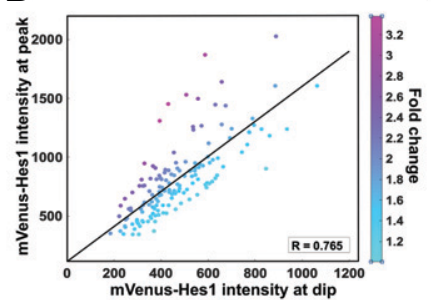

$\mathrm{E}$

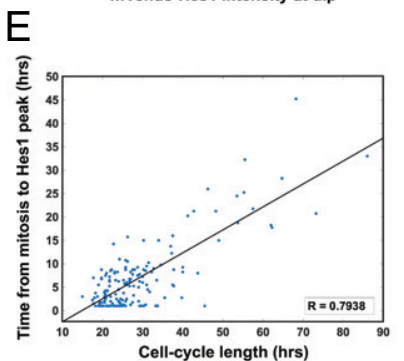

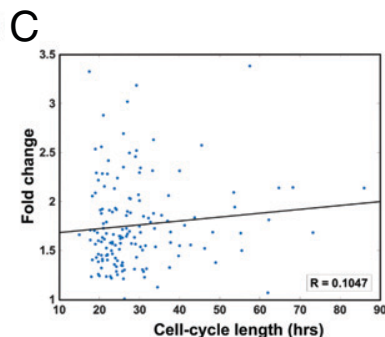

$\mathrm{F}$

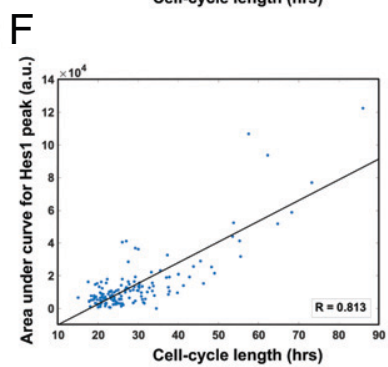

Fig. 7. The shape and the onset of the first Hes1 peak can predict the length of the cell cycle. (A) Example of a Hes1 trace with automatically detected peak of the Hes1 during G1 and the dip at or around G1-S transition. These Hes1 peak (maxima) and dip (minima) values were used to estimate fold-change in Hes1 expression when a cell transitions though the cell cycle. $(B)$ A strong correlation between Hes1 peak and dip values $(R=0.76)$ suggested that the Hes 1 fold-change values for cells remain within the same range (1.5to 2-fold), irrespective of whether a cell started with higher or lower Hes1 intensity. (C) When these estimated fold-change values were plotted against the cell-cycle lengths, no correlation was found $(R=0.10)$, showing that the cell-cycle lengths in the MCF-7 subpopulations were not influenced by the Hes 1 fold-change values. $(D)$ The same dip detection pipeline was used to estimate how long it took for a cell to reach the peak of Hes 1 expression after mitosis $(t)$, and also the AUC

for Hes1 expression during the first phase of the Hes1 expression. ( $E$ and $F$ ) Both time-to-peak and AUC showed strong positive correlations with the cellcycle lengths ( $R=0.78$ and 0.81 , respectively). These data shows that the shape and the onset of the Hes 1 expression in its first peak were the best predictors of the cell-cycle lengths in MCF-7 subpopulations. 
A
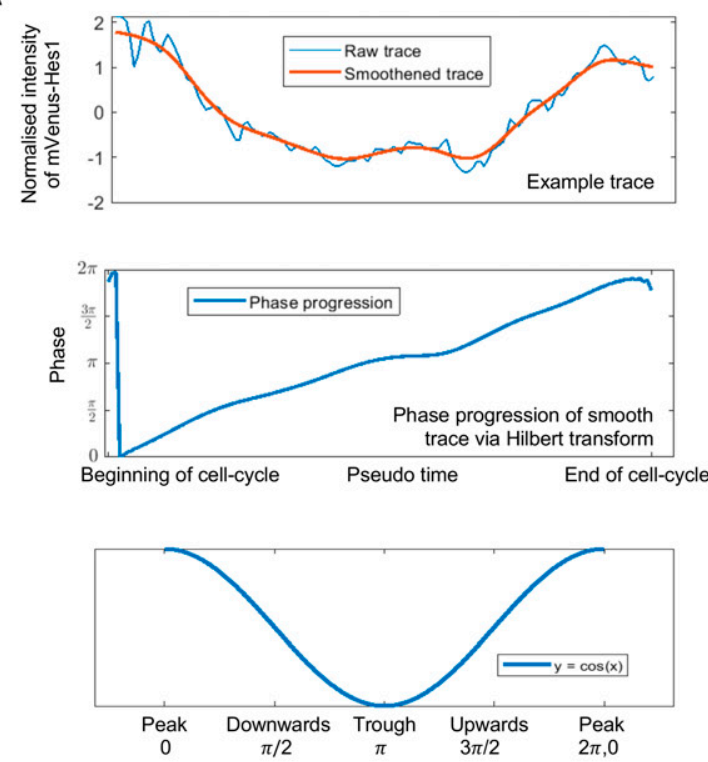

C

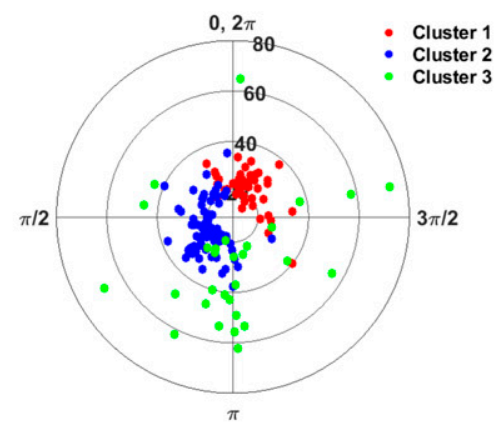

B
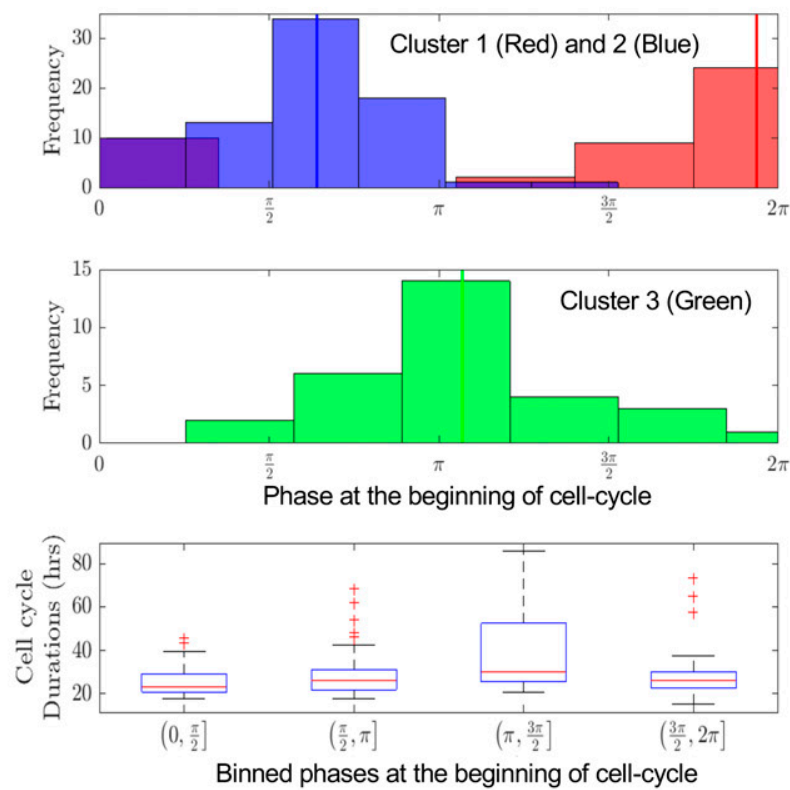

D
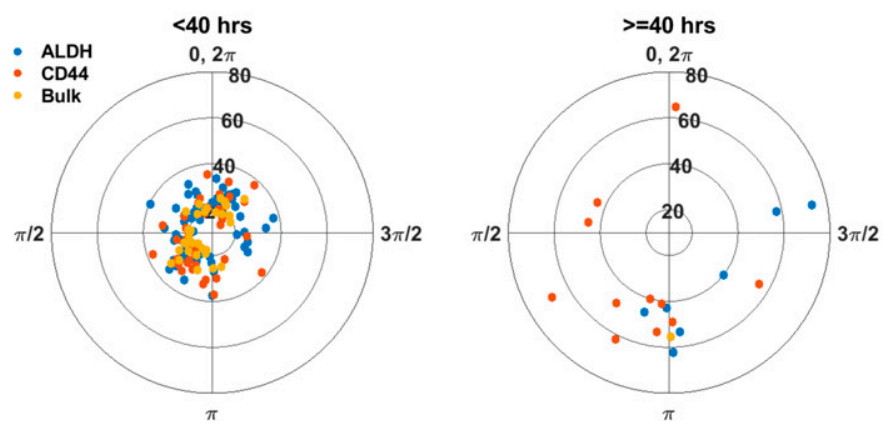

Fig. 8. The phase register of mitoses with the Hes1 oscillator and its influence on the length of the cell cycle; mitosis is phase-shifted in relation to the Hes1 oscillator in cells with the longer cell cycle. ( $A$, Top) An example of a normalized ( $z$-scored) Hes1 pseudotime series (blue) over one cell cycle (mitosis to mitosis) with smoothened trace (orange). (Middle and Bottom) Phase reconstruction for the smoothened trace (above) via Hilbert transform with phase readouts of an example cosine wave on the $x$ axis in the Bottom panel. Phases 0 or $2 \pi$ represent peak and phase $\pi$ represents a trough in the Hes 1 wave in one cell cycle. (B) Histograms showing where on the Hes1 wave, starting mitoses took place for cells from clusters 1 and 2 (Top) and cluster 3 (Middle) using the Bottom panel in $A$ as a reference. For cells from clusters 1 and 2, the majority of the mitoses took place around the descending or at the Hes 1 peak (Top). For cells from cluster 3, the majority of the mitoses happened at an apparent Hes 1 trough. These cells show a delayed Hes 1 peak in G1 which makes the starting level of Hes 1 at the exit of the previous mitosis appears retrospectively as a trough. (C) Scatter plot in polar coordinates where the angle (reading counterclockwise from the vertical) represents the phase read-out at the mitosis point preceding a cell cycle. The distance from the center of the circle represents the cell-cycle length of that cell. Cluster 1 cells are in red, cluster 2 in blue and cluster 3 in green. ( $D$ ) Scatter plots similar plot to $C$, except here ALDH ${ }^{\text {High }}$ bCSCs are in blue, CD44 ${ }^{\text {High }}$ CD $24^{\text {Low }}$ bCSCs are in orange and bulk cells are in yellow. The Left panel shows cells with cell-cycle lengths up to $40 \mathrm{~h}$, and the Right panel shows cells with cell-cycle lengths over $40 \mathrm{~h}$. Note that cluster 3 and CD44 ${ }^{\text {High }} \mathrm{CD} 24^{\text {Low }}$ cells, particularly the ones that have long cell cycles, preferentially occupy the lower half of the scatter plot (between $\pi / 2$ and $3 \pi / 2$ ), which suggests that the mitoses that give rise to these cells preferentially take place at or around a trough in the Hes 1 wave. Overall, histograms in $B$ and scatter plots in $C$ and $D$ show that preceding mitoses for cells with longer cell cycles (which mostly belong to cluster three and enriched for CD44 ${ }^{\text {High }}$ CD24 ${ }^{\text {Low }}$ bCSCs), register with the Hes1 wave out of phase (i.e., the mitoses occur around a trough), while cells with shorter cell cycles are more likely to divide around a peak of the Hes1 wave.

Hes1 expression dynamics. We made use of a viral expression system wherein mVenus-Hes1(cDNA) is expressed under the Ubc promoter (Ubc:mVenus-Hes1) for a sustained rather than a dynamic mVenus-Hes1 expression in the parental MCF-7 cells (Fig. $10 A-F$ ). MCF-7 cells expressing this reporter showed variability in the levels of the exogenous mVenus-Hes1 expression. To avoid any impact asserted by the overexpression of mVenus-Hes1, we focused on cells with low to medium expression levels of the exogenous mVenus-Hes1. Periodicity analysis of mVenus-Hes1 traces using the LSP platform and the heat maps generated from the normalized expression confirmed that the mVenus-Hes1 expression generated from this viral reporter was not periodic (Fig. $10 A$ and $B$ ). Any protein fluctuations were aperiodic and the characteristic Hes1 dip marking G1-to-S transition at a fixed position before mitosis was absent (Fig. $10 C$ and $D$ ). When these low-medium Ubc: mVenus-Hes1 expressers were compared to the control MCF-7 cells expressing nuclear Venus alone (Ubc:NuVenus), the experimental cells showed significantly longer cell-cycle lengths (Fig. 10E) and, overall, fewer cell divisions (Fig. 10F). Sustained expression of Hes1 did not only elongate the cell cycle but this was also accompanied by an increase in the proportion of CD $44^{\mathrm{High}} \mathrm{CD} 24^{\mathrm{Low}}$ cells (SI Appendix, Fig. S12); this may happen as a result of sustained Hes1 expression suppressing the switching behavior of $\mathrm{CD} 44^{\mathrm{High}} \mathrm{CD} 24^{\mathrm{Low}}$ cells. Thus, sustained Hes1 expression under a ubiquitous promoter alters 

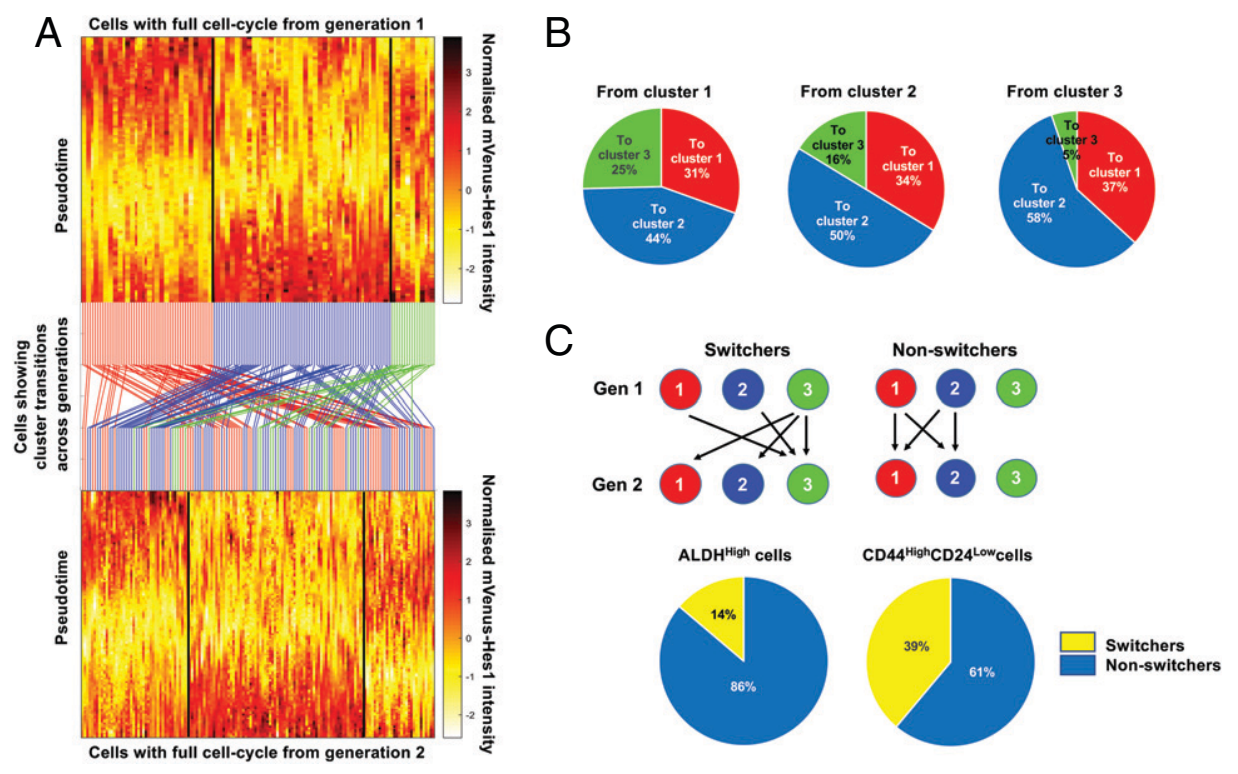

Fig. 9. Cells exhibit weighted cluster transitions, with $\mathrm{CD} 44^{\mathrm{High}} \mathrm{CD} 24^{\text {Low }}$ cells showing higher switching behaviors, compared to the ALDH ${ }^{\text {High }}$ cells. (A) Heatmaps of normalized ( $z$-scored) Hes1 pseudotime series with the full cell cycle classified as clusters in two consecutive generations (gen 1 Upper and gen 2 Lower). Cells across these two generations have been connected through lines (red, blue, green, corresponding to clusters 1,2 , and 3 as read from left to right) in the middle of these two heatmaps, showing how individual cells jump across clusters when they go from one to another generation. (B) Cluster transition rates show that these cluster transitions were not completely random, but weighted, with cluster 3 cells more likely to transition out into clusters 1 and 2 (Right pie chart), but clusters $1 / 2$ were more likely to stay within clusters $1 / 2$, and less likely to transition into cluster 3 (Left and Center pie charts). (C) Upper panel defines switchers as cells moving in or out of cluster 3, while nonswitchers as cells staying within clusters $1 / 2$, irrespective of their cell generations. $C D 44^{\text {High }} \mathrm{CD} 24^{\text {Low }}$ cells are more likely to switch (39\%) as compared to ALDH ${ }^{\text {High }}$ cells (only 14\%) (Lower), corroborating the previous findings that $\mathrm{CD} 44^{\mathrm{High}} \mathrm{CD} 24^{\mathrm{Low}}$ cells are more heterogenous in cell-cycle lengths.

cell-cycle length and cell fate in MCF-7 cells. Together, these data suggest that dynamics of Hes1 expression is required for smooth progression of the cell-cycle and cell-fate specification.

In the experiments above, to exclude the possibility that any effect of Ubc:Hes1 on cell cycle and cell fate is due to Hes1 overexpression above its physiological level, we transduced M18 cells (CRISPR/Cas9 line expressing mScarlett-Hes1) (Materials and Methods and SI Appendix, Fig. S4 $A-C$ ) with lentivirus expressing Ubc:mVenus-Hes1. We then compared the overall Hes1 fluorescence intensity in transduced stable lines with that of endogenously tagged M18 or C19 cells (SI Appendix, Fig. S13A). We found that the overall Hes1 intensity in transduced cells was very similar to mScarlet-Hes1 or mVenus-Hes1 intensity in untransduced M18 or Cl19 cells, respectively (SI Appendix, Fig. S13B). The collected mean intensities of mVenus and mScarlet were level-matched against each other for these analyses, as shown in SI Appendix, Fig. S13B .

To understand why the addition of Ubc:mVenus Hes1 expression does not raise the total Hes1 level, we looked at its impact on the endogenous Hes1 expression (tagged with mScarlet) and found an almost complete suppression of the endogenous expression (SI Appendix, Fig. S13 $A$ and $C$ ). We suggest that this is due to the property of autorepression of the Hes1 gene on its own promoter, which results in Ubc:mVenus Hes1 expression repressing the endogenous gene but not the other way around. We have made similar observations in mouse Hes1 in the context of neural stem cells (31). In conclusion, the suppression of endogenous Hes1 gene expression by exogenous mVenus-Hes1 driven off the Ubc promoter serves to maintain the total Hes1 at a physiological mean level, thus linking the phenotypic effects to altered dynamics rather than an altered mean level of expression.

Furthermore, to test for functional evidence of the reciprocal interaction between the cell cycle and the Hes1 oscillations, we inhibited cell-cycle progression by treating cells with $\mathrm{CoCl}_{2}$ (Materials and Methods) (32) and looked at Hes1 oscillations. We found that Hes1 oscillations continued even after the cellcycle was blocked but the periodicity was longer (Fig. 10G). These data suggest that the cell cycle and the Hes1 oscillator represent oscillations that can proceed independently with a longer "free running" period, but are normally coupled.
Sustained Hes1 Expression Impacts the Dynamics of the Cell-Cycle Regulators. To understand and strengthen the functional link between the Hes1 dynamics and the cell cycle, we made use of the mVenus-p21 CRISPR MCF-7 line generated using a similar CRISPR-tagging strategy (33). Using a single-cell, live-imaging approach, we monitored the expression of p21 under conditions of control and sustained Hes1 expression. We found that under control conditions, p21 had a peak of expression in the G1 phase, prior to the G1-S transition, as previously reported (33). But when we transfected the mVenus-p21 cells with a plasmid expressing sustained mScarlet-Hes1 under the Ubc promoter, p21 also lost its expression peak, showed sustained expression, and cells did not divide (SI Appendix, Fig. S14). These data suggest that Hes1 may interact with the cell-cycle machinery via its regulation of the dynamics of the key cell-cycle molecules, such as p21.

\section{Discussion}

The oscillatory protein expression of Hes1, a transcriptional repressor of the helix-loop-helix family, has been shown to be important for cell-state transitions in neural progenitor cells $(8$, $9,21)$. Even though such progenitor cells divide, it was an open question how Hes1 oscillations relate to the process of cell division. In this paper, we have asked whether Hes1 oscillations are observed in breast cancer cells and whether they have a reproducible and functional relation to their cell cycle.

Using an endogenous CRISPR/Cas9 fluorescent fusion reporter, we show that Hes1 oscillates with an average periodicity of $25 \mathrm{~h}$ in MCF-7 breast cancer cells. This is significantly longer than the previously reported ( 2 to $3 \mathrm{~h}$ ) ultradian periodicity in mouse cells (31, and references within). We did notice that similar ultradian oscillations are also "embedded" in the longer duration oscillations we report in this paper (as is apparent in the example Hes1 time trace shown in Fig. 4D); they have a periodicity of 5 to $6 \mathrm{~h}$ possibly due to the slower pace of oscillation in human cells, as reported for somitogenesis (20, 34). In this study, continuous live-imaging of 4- to 5-d duration, which far exceeded any previous live-imaging for Hes1 protein using mouse-based (or any other) experimental systems, uncovered these nonultradian, circadian-like oscillations of Hes1 that we focused on, as they showed clear correlations with both cell- 
A

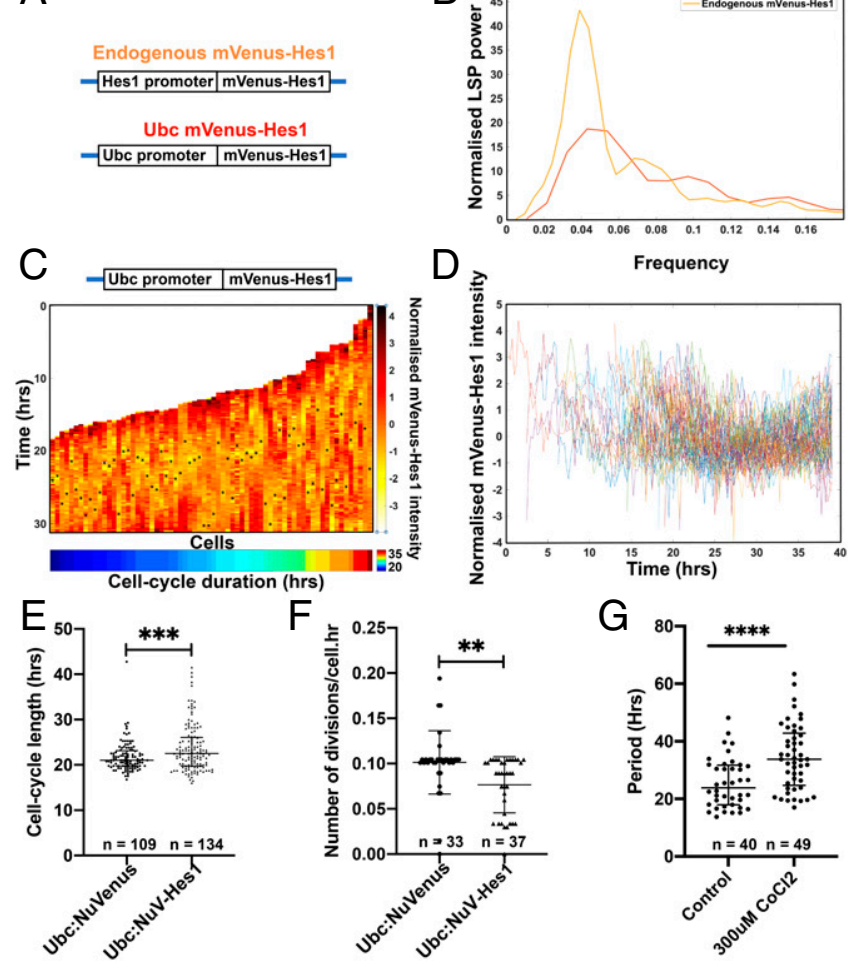

Fig. 10. Bidirectional interaction of Hes1 oscillations and the cell cycle. $(A-F)$ Dampening Hes1 oscillations by sustained expression of Hes1 elongates or impairs the cell cycle. $(A)$ Schematic of constructs for endogenous expression of mVenus-Hes1 (orange) and mVenus-Hes1 under the Ubc promoter (red). ( $B$ ) Normalized (scaled between 0 and 100) LSP power shows a strong peak in the power spectrum obtained from the endogenous $\mathrm{mVe}$ nus Hes 1 expression, in contrast to the mVenus-Hes 1 expression under the Ubc promoter. This suggested that endogenous mVenus-Hes 1 shows coherent oscillations while the same protein does not oscillate over time when expressed under the Ubc promoter. (C) Traces of Ubc-mVenus-Hes1 cells, normalized and arranged as a heatmap from mitosis to mitosis with time running vertically from top to bottom. These are aligned in time at the second mitosis of each cell. The time traces have been arranged in increasing order of the cell-cycle length. The dip in expression found by our ADD pipeline (black asterisk) shows random dips unlike in the Endogenous mVenus-Hes 1 time traces (Fig. $4 A$ and $D$ ). (D) Ubc-mVenus-Hes 1 time tracks from cells arranged as normalized time series traces from mitosis to mitosis, aligned in time at the ending mitosis, confirming the absence of a common dip in expression before division, compared to the endogenous mVenus-Hes1 as shown in Fig. $4 A$ and $D$. When Ubc:mVenus-Hes 1 cells were compared against control cells expressing $\mathrm{Nu}$-Venus alone for their cell-cycle properties, experimental cells were found to exhibit longer cell cycle $(* * * P=0.0005)(E)$ and to have lower number of cell divisions (**P= 0.0027) $(F)$. Together these data show that Hes1 oscillations are required for the smooth progression of the cell cycle. (G) Blocking the cell cycle using $\mathrm{CoCl}_{2}$, a hypoxia mimetic, elongates the periodicity of Hes1 (****P< 0.0001 ). This elongated Hes1 represents the period of Hes1 in the absence of an input from the cell cycle, possibly representing the free running period when Hes 1 oscillations and the cell cycle are not coupled. Control $n$ $=40$ cells, $300 \mu \mathrm{M} \mathrm{CoCl}_{2}, n=49$ cells.

cycle and cell fate, as compared to the ultradian oscillations (SI Appendix, Fig. S6).

MCF-7 cells are a good model to study nongenetic heterogeneity in breast cancer because they contain bulk cells: that is, cells with limited potential for tumor (re-)initiation, as well as populations of cells that can act as cancer stem cells when transplanted in vivo, namely ALDH ${ }^{\text {High }}$ epithelial-like stem cells and $\mathrm{CD} 44^{\mathrm{High}} \mathrm{CD} 24^{\text {Low }}$ mesenchymal-like stem cells (18). Here, we report that the majority of the MCF-7 cells (represented by bulk cells) have a consistent cell-cycle length of $\sim 24 \mathrm{~h}$, while cells sorted for known cancer stem cell markers show greater variability in their cell-cycle length. Interestingly, stem cells expressing $\mathrm{CD} 44^{\mathrm{High}} \mathrm{CD} 24^{\mathrm{Low}}$ markers, which are more likely to become dormant in vivo, show more significant variability than the average of the population and significantly longer cell cycles, which may predispose them to quiescence, in the right environment.

To understand how these long cell cycles may be generated, we asked whether the cell cycle interfaces with the Hes1 oscillations in a manner that may be causal. Indeed, we report here that, in most cells, Hes1 oscillations have a reproducible relationship to the cell cycle, such that mitosis tends to take place at or around the peak of Hes1 expression (in-phase register). Following mitosis, a trough of Hes1 expression precedes or coincides with the G1to-S transition. After this trough, Hes1 protein concentration picks up again as the cells progress to the second mitosis. Thus, because of the tendency of mitosis to take place around the peak, Hes1 expression appears biphasic within every cell cycle, with two periods of increased protein concentration in G1 and G2. This biphasic expression may reconcile conflicting activities of Hes1, which is known to repress both activators (such as CyD, CyE, and E2F) (14-16) and inhibitors of the cell-cycle (such as the CDKIs p21 and p27) (12,13), by providing a temporal separation of repressing phases with a period of de-repression at the trough. In fact, most of these proteins show dynamic expression during the cell cycle $(33,35)$ and we have shown that the expression of p21 is no longer dynamic when Hes1 is sustained, suggesting that Hes1 expression dynamics normally contributes in shaping or "sculpting" the dynamics of the cell-cycle molecules. Future experiments will show whether de-repression of molecules, such as E2-F1 or CyclinE (a target of Hes1 in MCF-7 cells and known for its function in G1-S transition, https://www.encodeproject.org/ experiments/ENCSR109ODF/), may be due to the dip in Hes1 expression and in turn may time the G1-S transition. The trough in Hes1 expression at around S-phase is most likely transcriptional, as it is not observed when Hes1 is expressed under a ubiquitous promoter and may be due to the loss of Notch signaling in S-phase, reported in developmental systems (36).

The observed variability in the cell-cycle length was specifically associated with the first peak of Hes1 expression during the cell cycle, while the time between the Hes1 trough in G1/S and the subsequent mitosis was less variable. This fits well with reports in the literature where in neural stem cells, the cellcycle length is primarily determined by the length of G1 (37). Given this, we further analyzed the expression of Hes1 in G1 phase of the cell cycle to see which Hes1 expression feature shows the best correlation with the cell-cycle length, implying a functional decoding role. One could hypothesize that cells that start with high Hes1 level would take longer to reach a set low Hes1 level, which could be required for G1-S. However, we found that neither the starting levels nor the mean Hes1 levels during the cell cycle showed a good correlation with cell-cycle length. Furthermore, the trough of Hes1 levels had a little varied fold-change in relation to the starting level, suggesting that there is no fixed absolute level of low Hes1 to which the dynamics tend. This finding implies that any downstream targets of Hes1 sense a fold-difference rather than an absolute minimum level of Hes1 at or around G1-S transition during the cell-cycle progression. Thus, Hes1 oscillations may be an underappreciated example of a fold-change detection system, a possible feature of gene regulatory networks with feedback (38).

Instead, we found that the position (onset) and the AUC (shape) of the first Hes1 expression peak, as recorded in liveimaging traces, was a better predictor of cell-cycle length than its levels of expression. Cells with a delayed onset Hes1 peak during the G1 phase of the cell cycle; that is, a Hes1 peak positioned with some temporal separation from the previous mitosis tended to have a longer cell cycle going forward. Such a delayed Hes1 peak in G1 supersedes the previous G2 peak, which is no longer a 
peak but part of an ascending Hes1 trend (picture abstract and example trace in Fig. $3 D-F$ ). Therefore, these cells are now out of phase with the cell cycle and the Hes1 period is elongated when one measures the distance from the delayed peak to the Hes1 peak in the preceding G1 (SI Appendix, Fig. S11B). Indeed, phase reconstruction showed that cells with longer cell cycles tended to have an unusual phase register with the cell cycle such that their mitoses tend to take place at around Hes1 trough rather than at the peak of Hes1 wave. This delayed Hes1 expression peak in G1 appears to have a knock-on effect in also delaying the trough, which if Hes1 has a gating effect, would delay the G1-to-S transition and the overall cell-cycle length of these cells (SI Appendix, Fig. S10B).

Furthermore, $\mathrm{CD} 44^{\mathrm{High}} \mathrm{CD} 24^{\mathrm{Low}}$ stem-like cells were more likely than $\mathrm{ALDH}^{\mathrm{High}}$ stem-like cells to show a late-onset Hes1 peak in G1 and, thus, a phase-shifted registration of Hes1 dynamics with the cell cycle. However, the phase registration was not set in time and cells belonging in different cluster classifications (with respect to their alignment of Hes1 dynamics with the cell cycle) switched between behaviors randomly but with weighted probabilities. CD $44^{\text {High }} \mathrm{CD} 24^{\text {Low }}$ cells showed the highest probability of switching Hes1 dynamics in and out of phase with the cell cycle, which fits well with the greater heterogeneity in cell-cycle length in these cells. Future experiments to understand the basis of the increased switching behavior of these cells will be important, as this property may underlie the higher propensity of these cells to become dormant in vivo (18).

To investigate further if Hes1 oscillations and the cell-cycle represent coupled systems, we misexpressed mVenus-Hes1 under a ubiquitous promoter (Ubc). In cells expressing this construct, Hes1 did not oscillate showing only random fluctuations in its expression. Since previous findings showed that under conditions of overexpression, high Hes1 levels are associated with cellular dormancy $(22,39,40)$, we have taken care to show that the level of Hes1 is comparable to physiological levels. In this population, we found a higher proportion of $\mathrm{CD} 44^{\mathrm{High}} \mathrm{CD} 24^{\mathrm{Low}}$ cells and a higher proportion of cells with a longer cell cycle or that failed to divide, suggesting that Hes1 oscillations are necessary for an efficient cell cycle with a consistent length. Reciprocally, blocking cell division completely with $\mathrm{CoCl}_{2}$, a hypoxia mimetic known for blocking the cell cycle and pushing cells into dormancy (32), elongated the Hes1 period and reduced their amplitude. These results suggest that the cell cycle and Hes1 oscillations represent periodic systems that can proceed independently but are normally reciprocally coupled, with a preferred, fixed phase relationship.

In conclusion, Hes1 expression appears heterogeneous in snapshots of $\mathrm{ER}^{+}$breast cancer cells but this is due to asynchronous temporal oscillations with a circadian-like periodicity. Our findings suggest that most likely through regulation of cell-cycle molecules, these Hes1 dynamics sculpt the cell cycle to an efficient nearly 24-h periodicity. Under normal conditions, it appears that cellcycle and Hes1 oscillations are coupled systems and their dynamic phase registration is important in controlling the cell-cycle length and cell fate. In that respect, our findings have some analogies to developmental systems, where the phase shift of periodic signaling molecule encodes information for cell fate (34). Notably, a particular subtype of stem-like cancer cell, the $\mathrm{CD} 44^{\mathrm{High}} \mathrm{CD} 24^{\mathrm{Low}}$ cells, show a higher rate of in- and out-of-phase switching and greater heterogeneity of the cell cycle than $\mathrm{ALDH}^{\mathrm{High}}$ cancer stem-like cells. This suggests that frequent misalignment of the Hes1 oscillations with the cell cycle may eventually contribute to the known tendency of $\mathrm{CD} 44^{\mathrm{High}} \mathrm{CD} 24^{\mathrm{Low}}$ cells to drop out of the cell cycle in vivo, which may have important implications for the progression and relapse of the breast disease. We suggest than an important area of future work is the interface of Hes1 with the circadian clock, which also shows coupling with the cell cycle (41) and has important implications for the chronobiology of cancer $(42,43)$.

\section{Materials and Methods}

Cell Culture. MCF-7 cells (from Manchester Breast Center) were maintained in high glucose DMEM plus $10 \%$ serum for all experiments, with the exception of cell-cycle arrest induction, which was achieved by adding $\mathrm{CoCl}_{2}$ (Merck, 255599) in the full growth media. Growth was assessed using an Incutyte livecell analysis system (Sartorius). MCF-7 cells were FACS-sorted to obtain defined subpopulations of cells using a FACSAria Fusion cell sorter (BD Biosciences) and appropriate antibodies (allophycocyanin-conjugated CD44/CD44-APC, Beckton Dickinson and phycoerythrin-conjugated CD24/CD24-PE, Beckman Coulter) for CD44 ${ }^{\text {High }}$ CD24 ${ }^{\text {Low }}$ cells or with AldeRed ALDH Detection Assay kit (Sigma) or ALDHFLOUR kit (StemCell Technologies) ALDH ${ }^{\text {High }}$ and ALDH ${ }^{\text {Low }}$ cells.

Reporter Lines (Endogenous and Viral). Reporter lines were created by $\mathrm{N}$-terminal in-frame fluorescent tagging (monomeric Venus/mVenus or monomeric Scarlet/mScarlet) of human endogenous Hes 1 gene in MCF-7 cells using CRISPR/Cas9 genome-editing technology. Clones were selected by FACS sorting for fluorescence, single-cell cloned, and genotyped. MCF-7 endogenous p21 reporter line were a gift from the Lahav laboratory, Harvard Medical School, Boston, MA (33). cDNA constructs for lentiviral transductions (Ubc:mVenus, Ubc:NuVenus, Ubc:mCherry-PCNA) were made using the pLNT vector. A similar lentiviral construct (pLV-Ubc:mVenus-Hes1) was obtained commercially from VectorBuilder.

Analysis of Gene and Protein Expression. Hes1 mRNA was quantitated by smFISH, as previously described (9), using a set of $32 \times 20$-nt probes against Human Hes1 exonic sequence, labeled with Quasar 570 (Biosearch Technologies) at their 5 ' end. Immunostainings were performed following standard protocols using either commercial HES1 primary antibody (E5 clone, sc-166410, Santa Cruz Biotechnology, on formaldehyde-fixed MCF-7 cells) or mouse anti-PCNA (M0879, Dako) for cell-cycle phases estimation, after smFISH protocol. For protein half-life estimation of exogenous HA-Hes1, MCF-7 cells transfected with pCS2-HA-Hes1 plasmid and treated with $100 \mathrm{uM} \mathrm{CHX} \mathrm{for} \mathrm{the} \mathrm{time} \mathrm{points} \mathrm{indi-}$ cated and analyzed by Western blot using a using HA-HRP antibody (Roche).

Data Collection Using Confocal Microscopy and Cell Tracking Using IMARIS. MCF-7 lines expressing fluorescent reporters were live-imaged every $15 \mathrm{~min}$ (unless stated otherwise) for 60 to $120 \mathrm{~h}$ using a Nikon A1R confocal microscope and the resulting $z$-stacked images were manually tracked using the "spot-tracking" function of the image analysis software IMARIS to obtain "mean" intensity profiles for Hes1 expression (in arbitrary units [a.u.]), representing changes in Hes1 "concentration" over time, in single cells and over (consecutive) cell divisions.

Data Analyses Methods and Custom Generated Codes. Data analyses of time series were performed with custom scripts on MATLAB. Hes 1 expression profiles were generated as time tracks and heat maps of $z$-score normalized Hes 1 intensity values. Pseudotime series of a constant length were generated using linear interpolation and distinct groups were obtained with Gaussian mixture clustering, using synthetic data as a control. We developed an ADD method for the normalized mVenus-Hes1 expression during the cell cycle by using a Savitzgy-Golay filter to smoothen the traces and identify the turning points. For periodicity analysis, we used a continuous-wavelet based time-frequency analysis using pyBOAT, LSP, autocorrelation, and ultradian periodicity analyses using a Gaussian process method, as indicated in the text. For phase diagrams, we applied the Hilbert transform onto smoothened normalized ( $z$-scored) mVenus-Hes1 pseudotime traces from mitosis to mitosis. To measure the strength and direction of a linear relation between two variables (such as Hes1 mean intensity and cell-cycle length), we used Spearman correlation coefficient plots. Statistical analyses were as indicated in the figure legends using Prism 8 (GraphPad) or Bartlett's test (through MATLAB) to check if the variances were equal across the groups of data. Errors bars are reported as SD of the mean (mean $\pm S D$ ).

For detailed information on this section, please refer to SI Appendix, Materials and Methods.

Data Availability. Examples of raw single-cell live-imaging files, excel files consisting of tracking information (Hes1 mean intensities, cell-cycle phase information), ICC images, and smFISH images have been deposited in Figshare, https://figshare.com/projects/Differential_phase_register_of_Hes1_oscillations_ with_mitoses_underlies_cell-cycle_heterogeneity_in_ER_breast_cancer_ cells_/119019.

ACKNOWLEDGMENTS. We thank Profs. Robert Clarke from the Manchester Breast Centre, and Keith Brennan from the Cell Matrix Centre, Faculty of Biology, Medicine, and Health (FBMH), University of Manchester, for 
providing the parental MCF-7 cells and the human Hes1 CDNA plasmids, respectively; Dr. Gareth Howell and Mr. Michael Jackson from the FACS facility, $\mathrm{FBMH}$, for all their help and expertise in sorting and analyzing the cell lines used in this study; Prof. Galit Lahav (Harvard Medical School) and Dr. Jacob Stewart-Ornstein (University of Pittsburgh) for their prompt

1. A. Raj, A. van Oudenaarden, Nature, nurture, or chance: Stochastic gene expression and its consequences. Cell 135, 216-226 (2008).

2. A. Brock, H. Chang, S. Huang, Non-genetic heterogeneity-A mutation-independent driving force for the somatic evolution of tumours. Nat. Rev. Genet. 10, 336-342 (2009).

3. P. B. Gupta, I. Pastushenko, A. Skibinski, C. Blanpain, C. Kuperwasser, Phenotypic plasticity: Driver of cancer initiation, progression, and therapy resistance. Cell Stem Cell 24, 65-78 (2019)

4. W. Chen, J. Dong, J. Haiech, M. C. Kilhoffer, M. Zeniou, Cancer stem cell quiescence and plasticity as major challenges in cancer therapy. Stem Cells Int. 2016, 1740936 (2016).

5. J. H. Levine, Y. Lin, M. B. Elowitz, Functional roles of pulsing. Science 342, 1193-1200 (2013).

6. A. Isomura, R. Kageyama, Ultradian oscillations and pulses: Coordinating cellular responses and cell fate decisions. Development 141, 3627-3636 (2014).

7. H. Hirata et al., Instability of Hes7 protein is crucial for the somite segmentation clock. Nat. Genet. 36, 750-754 (2004).

8. M. Goodfellow, N. E. Phillips, C. Manning, T. Galla, N. Papalopulu, microRNA input into a neural ultradian oscillator controls emergence and timing of alternative cell states. Nat. Commun. 5, 3399 (2014).

9. N. E. Phillips et al., Stochasticity in the miR-9/Hes1 oscillatory network can account for clonal heterogeneity in the timing of differentiation. eLife 5, 1-33 (2016).

10. X. Soto et al., Dynamic properties of noise and Her6 levels are optimized by miR-9, allowing the decoding of the Her6 oscillator. EMBO J. 39, e103558 (2020).

11. C. S. Manning et al., Quantitative single-cell live imaging links HES5 dynamics with cell-state and fate in murine neurogenesis. Nat. Commun. 10, 2835 (2019).

12. P. Castella, S. Sawai, K. Nakao, J. A. Wagner, M. Caudy, HES-1 repression of differentiation and proliferation in PC12 cells: role for the helix 3-helix 4 domain in transcription repression. Mol. Cell. Biol. 20, 6170-6183 (2000).

13. K. Murata et al., Hes1 directly controls cell proliferation through the transcriptional repression of p27Kip1. Mol. Cell. Biol. 25, 4262-4271 (2005)

14. C. Cenciarelli et al., The interference of Notch1 target Hes1 affects cell growth, differentiation and invasiveness of glioblastoma stem cells through modulation of multiple oncogenic targets. Oncotarget 8, 17873-17886 (2017).

15. N. Noda, S. Honma, Y. Ohmiya, Hes 1 is required for contact inhibition of cell proliferation in 3T3-L1 preadipocytes. Genes Cells 16, 704-713 (2011).

16. J. Hartman et al., HES-1 inhibits $17 \beta$-estradiol and heregulin- $\beta 1$-mediated upregulation of E2F-1. Oncogene 23, 8826-8833 (2004).

17. H. Pan et al., EBCTCG, 20-year risks of breast-cancer recurrence after stopping endocrine therapy at 5 years. N. Engl. J. Med. 377, 1836-1846 (2017).

18. S. Liu et al., Breast cancer stem cells transition between epithelial and mesenchymal states reflective of their normal counterparts. Stem Cell Reports 2, 78-91 (2013).

19. T. Kobayashi et al., Deubiquitinating enzymes regulate Hes1 stability and neuronal differentiation. FEBS J. 282, 2411-2423 (2015)

20. T. Rayon et al., Species-specific pace of development is associated with differences in protein stability. Science 369, eaba7667 (2020).

21. S. Ochi, Y. Imaizumi, H. Shimojo, H. Miyachi, R. Kageyama, Oscillatory expression of Hes1 regulates cell proliferation and neuronal differentiation in the embryonic brain. Development 147, dev182204(2020).

22. R. Sueda, R. Kageyama, Regulation of active and quiescent somatic stem cells by Notch signaling. Dev. Growth Differ. 62, 59-66 (2020). responses and for making available their MCF-7 p21 CRIPSR line; the Genomics facility, FBMH, for their sequencing services; and Dr. Anthony Adamson, from the Genome Editing Unit, $\mathrm{FBMH}$, for his guidance on the CRISPR/Cas9 designing and implementation. The work was sponsored by the Wellcome Trust Grant 106185/Z/14/Z (to N.P.).

23. R. Sueda, I. Imayoshi, Y. Harima, R. Kageyama, High Hes1 expression and resultan Ascl1 suppression regulate quiescent vs. active neural stem cells in the adult mouse brain. Genes Dev. 33, 511-523 (2019).

24. R. Kageyama, H. Shimojo, T. Ohtsuka, Dynamic control of neural stem cells by bHLH factors. Neurosci. Res. 138, 12-18 (2019).

25. T. Kobayashi, R. Kageyama, Expression dynamics and functions of Hes factors in development and diseases. Curr. Top. Dev. Biol. 110, 263-283 (2014).

26. G. A. Moenke, F.A. Sorgenfrei, C. Schmal, Optimal time frequency analysis for biolog ical data-pyBOAT. bioRxiv [Preprint] (2020). https://www.biorxiv.org/content/10. 1101/2020.04.29.067744v1 (Accessed 5 June 2020).

27. J. T. VanderPlas, Understanding the Lomb-Scargle Periodogram. (The Astrophysical Journal Supplement Series, 2018), vol. 236.

28. P. Stoica, R. Moses, Spectral Analysis of Signals (Prentice Hall, Upper Saddle River, NJ, 1997).

29. N. E. Phillips, C. Manning, N. Papalopulu, M. Rattray, Identifying stochastic oscilla tions in single-cell live imaging time series using Gaussian processes. PLOS Comput. Biol. 13, e1005479 (2017).

30. L. Leung, A. V. Klopper, S. W. Grill, W. A. Harris, C. Norden, Apical migration of nucle during $\mathrm{G} 2$ is a prerequisite for all nuclear motion in zebrafish neuroepithelia. Development 138, 5003-5013 (2011).

31. E. Marinopoulou et al., HES1 protein oscillations are necessary for neural stem cells to exit from quiescence. iScience 24, 103198 (2021).

32. H. R. Lee, F. Leslie, S. M. Azarin, A facile in vitro platform to study cancer cel dormancy under hypoxic microenvironments using $\mathrm{CoCl}_{2}$. J. Biol. Eng. 12, 12 (2018)

33. J. Stewart-Ornstein, G. Lahav, Dynamics of CDKN1A in single cells defined by an endogenous fluorescent tagging toolkit. Cell Rep. 14, 1800-1811 (2016).

34. K. F. Sonnen et al., Modulation of phase shift between Wnt and Notch signaling oscillations controls mesoderm segmentation. Cell 172, 1079-1090.e12 (2018).

35. A. R. Barr, F. S. Heldt, T. Zhang, C. Bakal, B. Novák, A dynamical framework for the allor-none G1/S transition. Cell Syst. 2, 27-37 (2016)

36. F. A. Carrieri et al., CDK1 and CDK2 regulate NICD1 turnover and the periodicity of the segmentation clock. EMBO Rep. 20, e46436 (2019)

37. N. Sabherwal, R. Thuret, R. Lea, P. Stanley, N. Papalopulu, aPKC phosphorylates p27Xic1, providing a mechanistic link between apicobasal polarity and cell-cycle control. Dev. Cell 31, 559-571 (2014)

38. O. Shoval et al., Fold-change detection and scalar symmetry of sensory input fields. Proc. Natl. Acad. Sci. U.S.A. 107, 15995-16000 (2010).

39. L. Sang, H. A. Coller, J. M. Roberts, Control of the reversibility of cellular quiescence by the transcriptional repressor HES1. Science, 1095-1100 (2008).

40. L. Sang, J. M. Roberts, H. A. Coller, Hijacking HES1: How tumors co-opt the antidifferentiation strategies of quiescent cells. Trends Mol. Med. 16, 17-26 (2010).

41. C. Feillet, G. T. J. van der Horst, F. Levi, D. A. Rand, F. Delaunay, Coupling between the circadian clock and cell cycle oscillators: Implication for healthy cells and malignant growth. Front. Neurol. 6, 96 (2015).

42. P. F. Innominato, F. A. Lévi, G. A. Bjarnason, Chronotherapy and the molecular clock: Clinical implications in oncology. Adv. Drug Deliv. Rev. 62, 979-1001 (2010).

43. P. F. Innominato et al., The circadian timing system in clinical oncology. Ann. Med. 46, 191-207 (2014). 